\title{
ASIFT: An Algorithm for Fully Affine Invariant Comparison
}

\author{
Guoshen $\mathrm{Yu}^{1}$, Jean-Michel Morel ${ }^{2}$ \\ ${ }^{1}$ CMAP, École Polytechnique, France (yu@cmap.polytechnique.fr) \\ ${ }^{2}$ CMLA, ENS Cachan, France (moreljeanmichel@gmail.com) \\ Communicated by Guillermo Sapiro Demo edited by Nicolas Limare
}

\begin{abstract}
If a physical object has a smooth or piecewise smooth boundary, its images obtained by cameras in varying positions undergo smooth apparent deformations. These deformations are locally well approximated by affine transforms of the image plane. In consequence the solid object recognition problem has often been led back to the computation of affine invariant image local features. The similarity invariance (invariance to translation, rotation, and zoom) is dealt with rigorously by the SIFT method The method illustrated and demonstrated in this work, AffineSIFT (ASIFT), simulates a set of sample views of the initial images, obtainable by varying the two camera axis orientation parameters, namely the latitude and the longitude angles, which are not treated by the SIFT method. Then it applies the SIFT method itself to all images thus generated. Thus, ASIFT covers effectively all six parameters of the affine transform.
\end{abstract}

\section{Source Code}

The source code (ANSI C), its documentation, and the online demo are accessible at the IPOL web page of this article ${ }^{1}$.

Keywords: SIFT; affine invariant matching

\section{Overview}

If a physical object has a smooth or piecewise smooth boundary, its images obtained by cameras in varying positions undergo smooth apparent deformations. These deformations are locally well approximated by affine transforms of the image plane.

In consequence the solid object recognition problem has often been led back to the computation of affine invariant image local features. Such invariant features could be obtained by normalization methods, but no fully affine normalization method exists for the time being. Yet as shown in [7] the similarity invariance (invariance to translation, rotation, and zoom) is dealt with rigorously by the SIFT method [1]. By simulating on both images zooms out and by normalizing translation and

\footnotetext{
${ }^{1}$ https://doi.org/10.5201/ipol.2011.my-asift
} 
rotation, the SIFT method succeeds in being fully invariant to four out of the six parameters of an affine transform.

The method illustrated and demonstrated in this work, Affine-SIFT (ASIFT), simulates a set of sample views of the initial images, obtainable by varying the two camera axis orientation parameters, namely the latitude and the longitude angles, which are not treated by the SIFT method. Then it applies the SIFT method itself to all images thus generated. Thus, ASIFT covers effectively all six parameters of the affine transform. The method is mathematically proved in [6] to be fully affine invariant. And, against any prognosis, simulating a large enough set of sample views depending on the two camera orientation parameters is feasible with no dramatic computational load. The main anamorphosis (deformation) from an image to another caused by applying affine transforms can be measured by the transition tilt, a new geometric concept introduced in [6] and explained below.

While state-of-the-art methods before ASIFT hardly exceeded transition tilts of 2 (SIFT), 2.5 (Harris-Affine and Hessian-Affine [3]) and 10 (MSER [2]), ASIFT can handle transition tilts up 32 and higher. MSER can actually deal with transition tilts as high as 10 only when both objects are taken roughly at the same distance. Indeed, contrarily to SIFT, MSER is not scale invariant, because it does not simulate the blur due to an increasing distance to the object. The affine transforms considered in the celebrated comparison paper [4] do not have high transition tilts as those that can be handled by ASIFT. As shown by the experiment archive, most scenes with negligible or moderate camera view angle change that match with ASIFT also match with SIFT (with usually fewer matching points). But, when the view angle change becomes important, SIFT and other methods fail while ASIFT continues to work, as we shall see in the examples (Section 6).

\section{Disclaimer}

The present work publishes only the ASIFT algorithm as described below. It does not publish the SIFT and ORSA subroutines which are called by the ASIFT code. SIFT and ORSA may be later updated or replaced by other subroutines.

\section{Affine Camera Model}

\subsection{Image Acquisition Model}

As illustrated by the camera model in Figure 1, digital image acquisition of a flat object can be described as

$$
\mathbf{u}=\mathbf{S}_{1} G_{1} A \mathcal{T} u_{0}
$$

where $\mathbf{u}$ is a digital image and $u_{0}$ is an (ideal) infinite resolution frontal view of the flat object. The parameters $\mathcal{T}$ and $A$ are respectively a plane translation and a planar projective map due to the camera motion. $G_{1}$ is a Gaussian convolution modeling the optical blur, and $\mathbf{S}_{1}$ is the standard sampling operator on a regular grid with mesh 1 . The Gaussian kernel is assumed to be broad enough to ensure no aliasing by the 1-sampling, therefore with a Shannon-Whittaker interpolation $I$, one can recover the continuous image from its discrete version: $I \mathbf{S}_{1} G_{1} A \mathcal{T} u_{0}=G_{1} A \mathcal{T} u_{0}$. $\mathbf{S}_{1}$ will be thus omitted in the following.

\subsection{Affine Local Approximation}

We shall proceed to a further simplification of the above model, by reducing $A$ to an affine map. Figure 2 shows one of the first perspectively correct Renaissance paintings by Paolo Uccello. The 


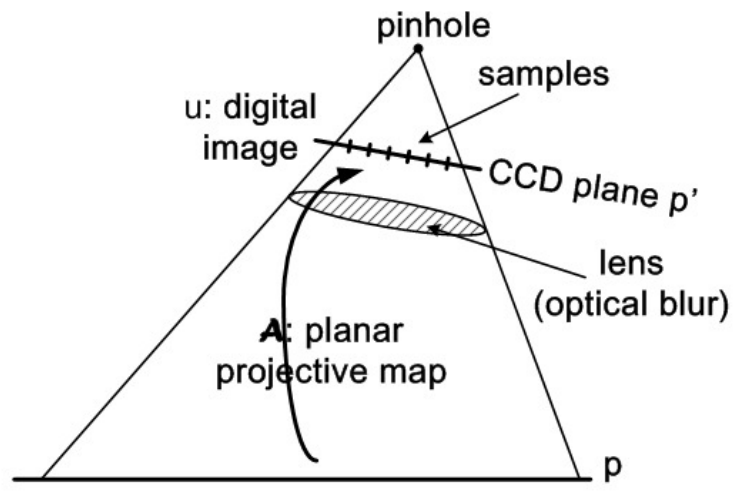

$\mathrm{u}_{0}$ : frontal infinite resolution view

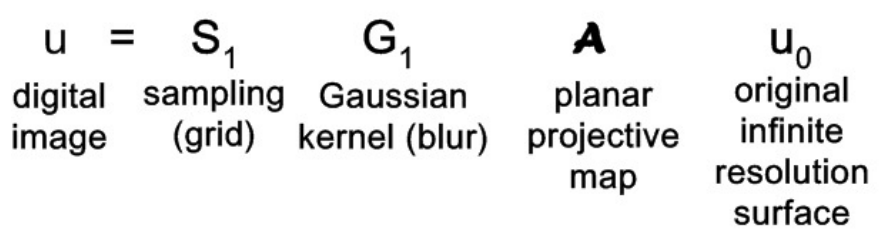

Figure 1: The projective camera model.

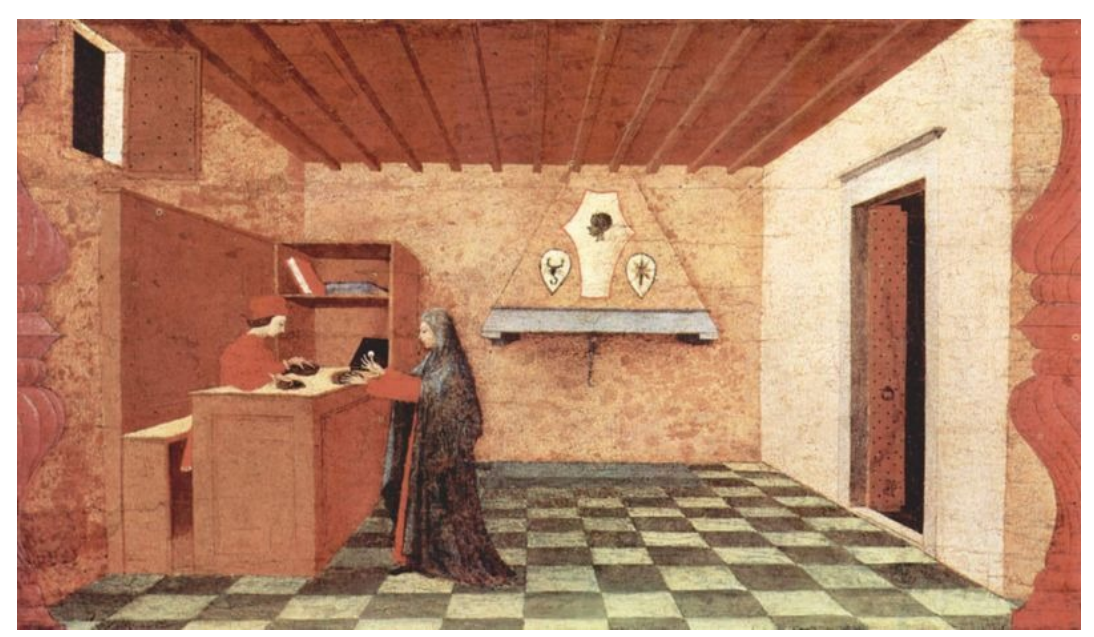

Figure 2: Affine local approximation.

perspective on the ground is strongly projective: the rectangular pavement of the room becomes a trapezoid. However, each tile on the pavement is almost a parallelogram. This illustrates the local tangency of perspective deformations to affine maps. Indeed, by the first order Taylor formula, any planar smooth deformation can be approximated around each point by an affine map. The perspective deformation of a plane object induced by a camera motion is a planar homographic transform, which is smooth, and therefore locally tangent to affine transforms $u(x, y) \mapsto u(a x+b y+e, c x+d y+f)$ in each image region.

\subsection{Affine Map Decomposition, with Geometric Interpretation as a Cam- era Motion}

Any affine map $A$ with strictly positive determinant which is not a similarity has a unique decomposition 


$$
A=\left[\begin{array}{ll}
a & b \\
c & d
\end{array}\right]=H_{\lambda} R_{1}(\psi) T_{t} R_{2}(\phi)=\lambda\left[\begin{array}{cc}
\cos \psi & -\sin \psi \\
\sin \psi & \cos \psi
\end{array}\right]\left[\begin{array}{ll}
t & 0 \\
0 & 1
\end{array}\right]\left[\begin{array}{cc}
\cos \phi & -\sin \phi \\
\sin \phi & \cos \phi
\end{array}\right],
$$

where $\lambda>0, \lambda t$ is the determinant of $A, R_{i}$ are rotations, $\phi \in[0, \Pi)$, and $T_{t}$ is a tilt, namely a diagonal matrix with first eigenvalue $t>1$ and the second one equal to 1 .

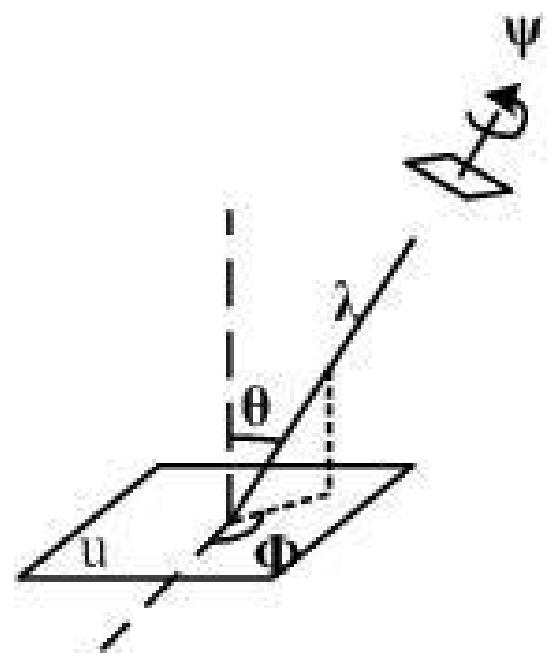

Figure 3: Geometric interpretation of affine decomposition.

Figure 3 shows a camera motion interpretation of the affine decomposition: $\phi$ and $\theta=\arccos 1 / t$ are the viewpoint angles, $\psi$ parameterizes the camera spin and $\lambda$ corresponds to the zoom. The camera (the small parallelogram on the top-right) is assumed to stay far away from the image $u$ and starts from a frontal view $u$, i.e., $\lambda=1, t=1, \phi=\psi=0$. The camera can first move parallel to the object's plane: this motion induces a translation $\mathcal{T}$ that is eliminated by assuming without loss of generality that the camera axis meets the image plane at a fixed point. The plane containing the normal and the optical axis makes an angle $\phi$ with a fixed vertical plane. This angle is called "longitude". Its optical axis then makes a $\theta$ angle with the normal to the image plane $u$. This parameter is called "latitude". Both parameters are classical coordinates on the "observation hemisphere". The camera can rotate around its optical axis (rotation parameter $\psi$ ). Last but not least, the camera can move forward or backward, as measured by the zoom parameter $\lambda$. We have seen that the affine model is enough to give an account of local projective deformations. If the camera were not assumed to be far away, the plane image deformation under a camera motion would be a homography. Yet, as explained above, a homography is locally tangent to an affine map.

\subsection{Transition Tilts, why can they be so High?}

The parameter $t$ defined above is called absolute tilt, since it measures the tilt between the frontal view and a slanted view. In real applications, both compared images are usually slanted views. The transition tilt is designed to quantify the amount of tilt between two such images. Assume that $v(x, y)=u(A(x, y))$ and $w(x, y)=u(B(x, y))$ are two slanted views of a flat scene whose image is $u(x, y)$, where $A$ and $B$ are two affine maps. Then $v(x, y)=w\left(A B^{-1}(x, y)\right)$. The transition tilt between $v$ and $w$ is defined as the absolute tilt associated with the affine map $A B^{-1}$. Let $t$ and $t^{\prime}$ the absolute tilts of two images $u$ and $u^{\prime}$, and let $\phi$ and $\phi^{\prime}$ be their longitude angles. The transition tilt $\tau\left(u, u^{\prime}\right)$ between the two images depends on the absolute tilts and the longitude angles, and satisfies

$$
t / t^{\prime} \leq \tau\left(u, u^{\prime}\right)=\tau\left(u^{\prime}, u\right) \leq t t^{\prime}
$$


where we assume $t \geq t^{\prime}$. The transition tilt can therefore be much higher than an absolute tilt. Hence, it is important for an image matching algorithm to be invariant to high transition tilts.

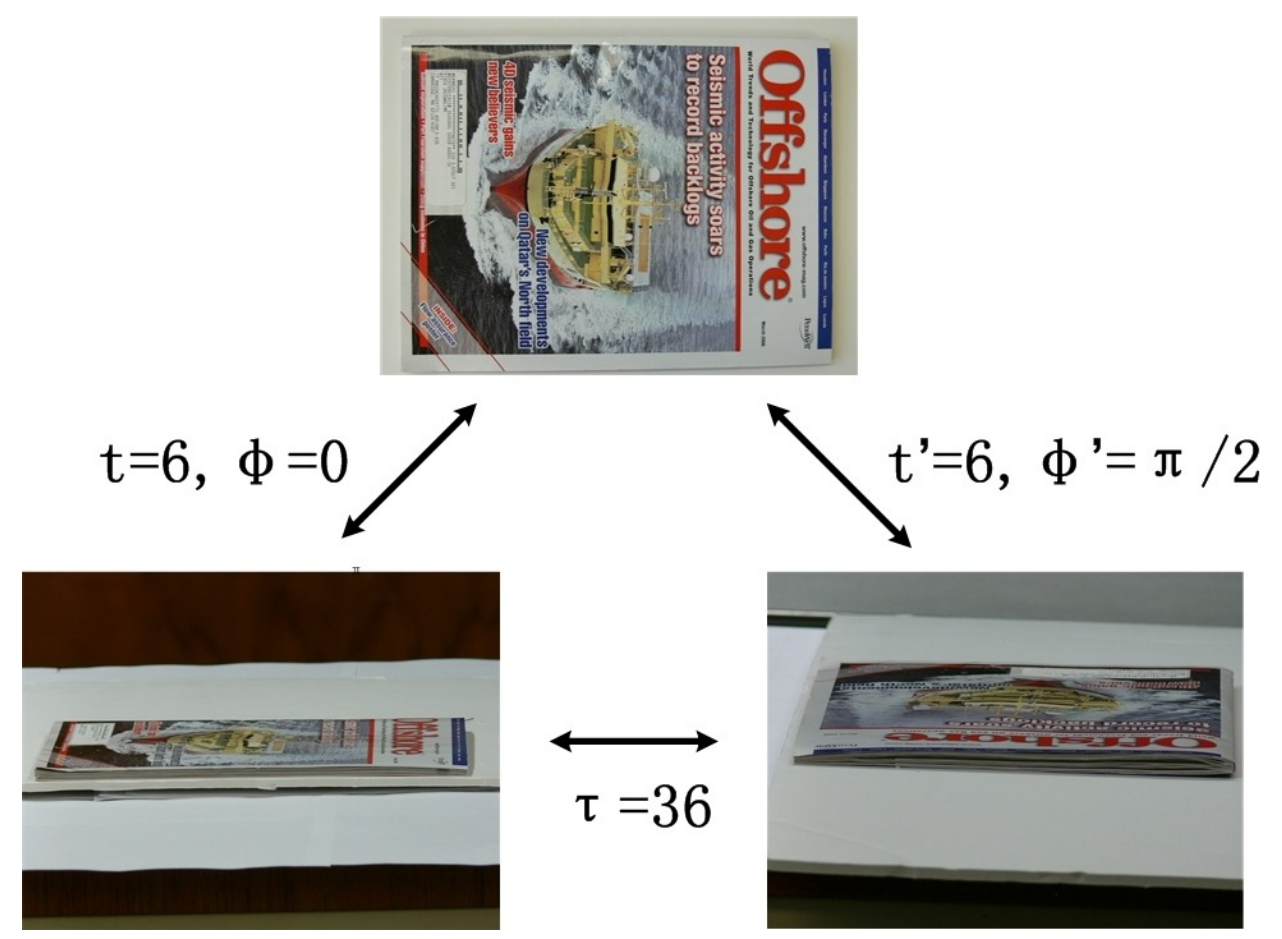

Figure 4: High transition tilt.

Figure 4 illustrates an example of high transition tilt. The frontal image (above) is squeezed in one direction on the left image by a slanted view, and squeezed in an orthogonal direction by another slanted view. The absolute tilt (compression factor) is about 6 in each view. The resulting compression factor, or transition tilt from left to right is actually $6 \times 6=36$.

\section{Description of the ASIFT Algorithm}

A fully affine invariant image matching algorithm needs to cover the 6 affine parameters. The SIFT method covers 4 parameters by normalizing rotations and translations, and simulating all zooms out of the query and of the search images.

As illustrated in Figure 5, ASIFT complements SIFT by simulating the two parameters that model the camera optical axis direction (the original and simulated images are represented respectively by squares and parallelograms), and then applies the SIFT method to compare the simulated images, so that all the 6 parameters are covered. In other words, ASIFT simulates three parameters: the scale, the camera longitude angle and the latitude angle (which is equivalent to the tilt) and normalizes the other three (translation and rotation). ASIFT can thus be mathematically shown to be fully affine invariant [6]. Against any prognosis, simulating the whole affine space is not prohibitive at all with the proposed affine space sampling $[6,8]$.

\subsection{Algorithm Description}

ASIFT proceeds by the following steps.

1. Each image is transformed by simulating all possible affine distortions caused by the change of camera optical axis orientation from a frontal position. These distortions depend upon two 


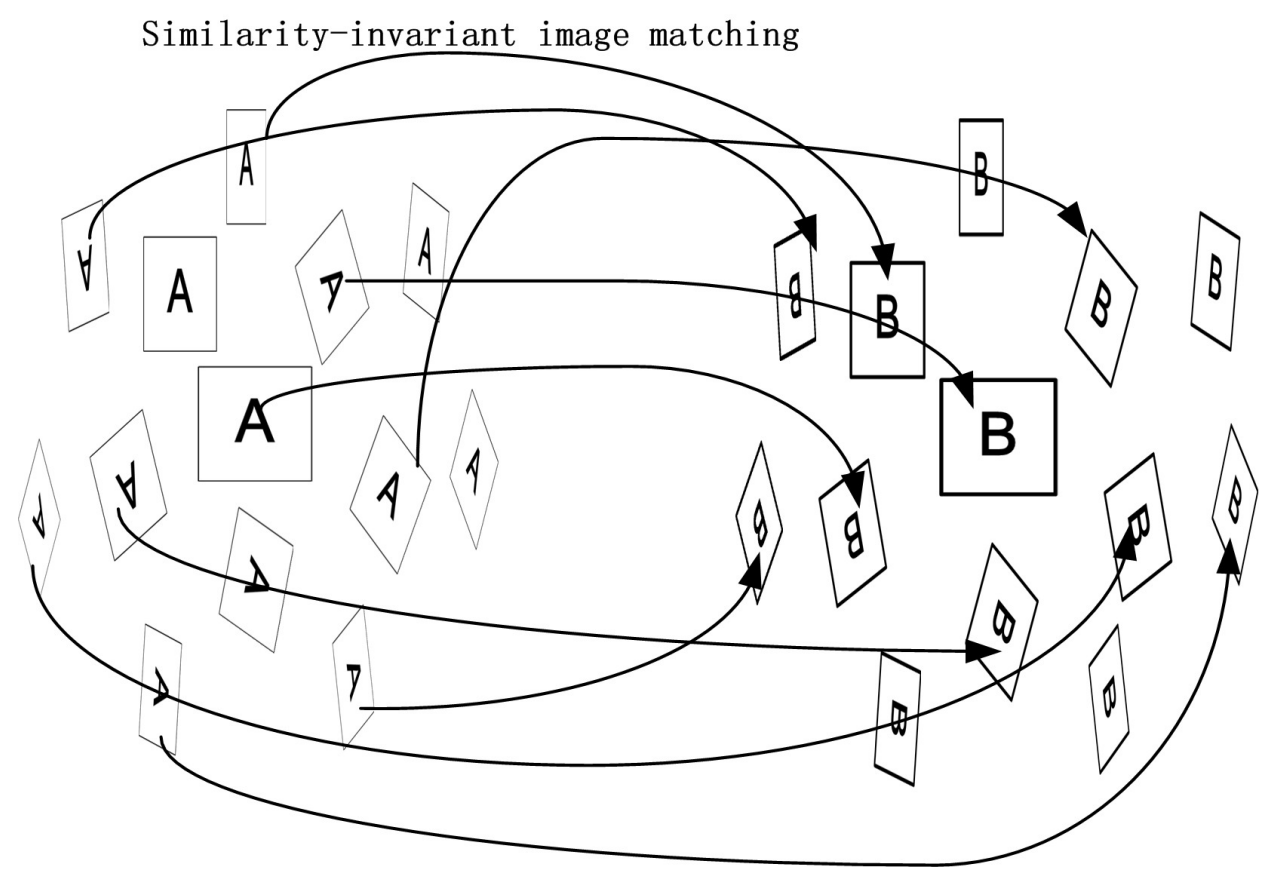

Figure 5: ASIFT algorithm overview.

parameters: the longitude $\phi$ and the latitude $\theta$. The images undergo rotations with angle $\phi$ followed by tilts with parameter $t=1 /|\cos \theta|$ (a tilt by $t$ in the direction of $x$ is the operation $u(x, y) \rightarrow u(t x, y))$. For digital images, the tilt is performed by a directional $t$-subsampling. It therefore requires the previous application of an antialiasing filter in the direction of $x$, namely the convolution by a Gaussian with standard deviation $\mathbf{c} \sqrt{t^{2}-1}$. The value $\mathbf{c}=0.8$ is the value shown [7] to ensure a very small aliasing error. These rotations and tilts are performed for a finite and small number of latitude and longitude angles, the sampling steps of these parameters ensuring that the simulated images keep close to any other possible view generated by other values of $\phi$ and $\theta$ (see below).

2. All simulated images are compared by a similarity invariant matching algorithm (SIFT). SIFT can be replaced by any other similarity invariant matching method. (There are many such variants of SIFT). It is therefore not the object of this article to describe the SIFT method.

3. The SIFT method has its own wrong match elimination criterion. Nonetheless, it generally leaves behind false matches, even in image pairs that do not correspond to the same scene. ASIFT, by comparing many pairs, can therefore accumulate many wrong matches. It is important to filter out these matches. The criterion used is that the retained matches must be compatible with an epipolar geometry. We use to that goal the ORSA method [5], which is considered the most reliable method, robust to more outliers than a classic RANSAC procedure. It is not the goal of this article to present ORSA. It is simply used to filter out the matches given by both SIFT and ASIFT. Thus, it may occur that two images have no match left at all. This does not necessarily mean that there are no ASIFT matches; the matches may be all eliminated as incompatible with an epipolar geometry.

\subsection{Parameter Sampling}

The sampling precision of the latitude and longitude angles should increase with $\theta$, since the image distortion caused by a fixed latitude or longitude angle displacement is more drastic at larger $\theta$. As 


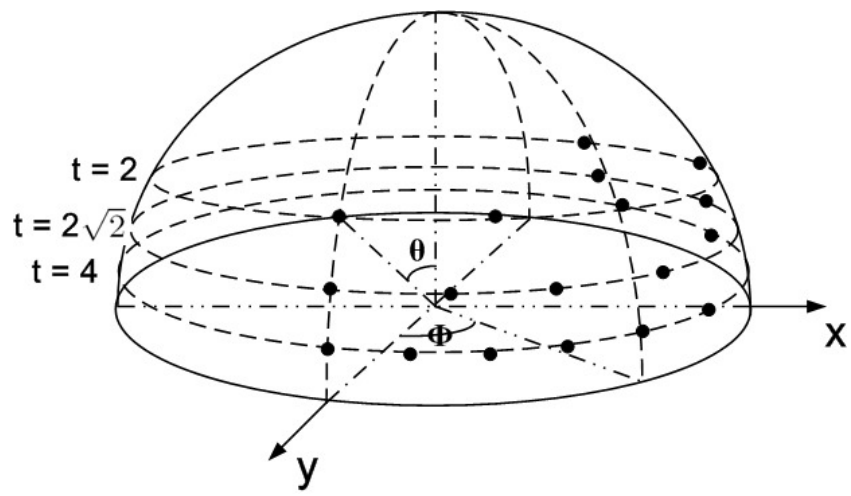

(a) Perspective illustration of the observation hemisphere.

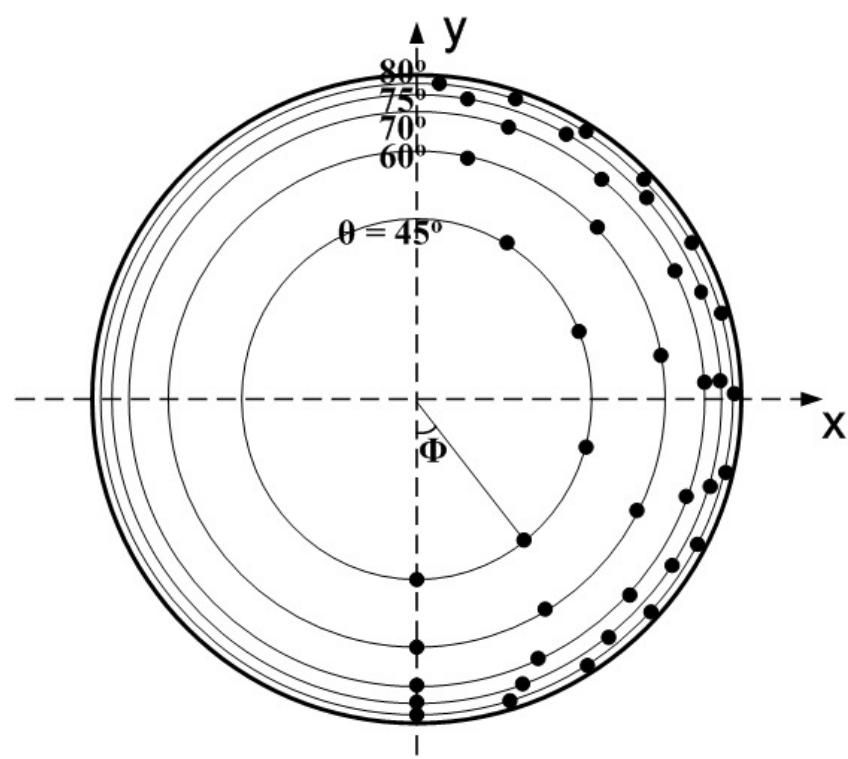

(b) Zenith view of the observation hemisphere.

Figure 6: Sampling of the parameters. The samples are the black dots.

described in Figure 6, the sampling of the latitude and longitude angles is specified below.

- The latitudes $\theta$ are sampled so that the associated tilts follow a geometric series $1, a, a^{2}, \ldots, a^{n}$, with $a>1$. The choice $a=\sqrt{2}$ is a good compromise between accuracy and sparsity. In the present implementation, the value $n$ goes up to 5 . In consequence transition tilts going up to 32 (and even a little bit more) can be explored.

- The longitudes $\phi$ are for each tilt an arithmetic series $0, b / t, \ldots, k b / t$, where $b \simeq 72^{\circ}$ seems again a good compromise, and $k$ is the last integer such that $k b / t<180^{\circ}$.

\subsection{Computational Complexity}

Estimating the ASIFT complexity boils down to calculating the image area simulated by ASIFT. The complexity of the ASIFT feature computation is proportional to the image area under test. With the parameter sample steps $\Delta t=\sqrt{2}, \Delta \phi=72^{\circ} / t$, as proposed above, the simulated image area is proportional to the number of tilts that are simulated. With the proposed simulated tilt range $\left[t_{\min }, t_{\max }\right]=[1,4 \sqrt{2}]$, which allows to cover a transition tilt as high as 32 , the total simulated area is about 13.5 times the area of the original image. The ASIFT feature computation complexity is therefore 13.5 times the complexity for computing SIFT features. The complexity growth is "linear" and thus marginal with respect to the "exponential" growth of transition tilt invariance.

Since ASIFT simulates 13.5 times the area of the original images, it generates about 13.5 times more features on both the query and the search images. The complexity of ASIFT feature comparison is therefore $13.5^{2} \simeq 180$ times as much as that of SIFT.

Note that on typical images, the ASIFT feature computation dominates the computational complexity of feature comparison. So the total ASIFT computation complexity typically is about 13.5 times that of SIFT when comparing only a few images. If instead the problem is to compare an image to a huge database, this complexity is no more negligible, and having 180 times more comparisons to perform is a serious limitation. 


\subsubsection{Coarse-to-fine Acceleration}

An easy coarse-to-fine acceleration described in [6] reduces respectively the complexity of ASIFT feature computation and comparison to 1.5 and 2.25 times that of SIFT. This acceleration is not used here.

\subsubsection{Parallelization}

In addition, the SIFT subroutines (feature computation and comparison) in ASIFT are independent and can easily be implemented in parallel. The online demo uses this possibility.

\section{Implementation}

\subsection{ASIFT Feature Computation}

Algorithm 1. Implemented in the $\mathrm{C}++$ source file compute_asift_keypoints.cpp.

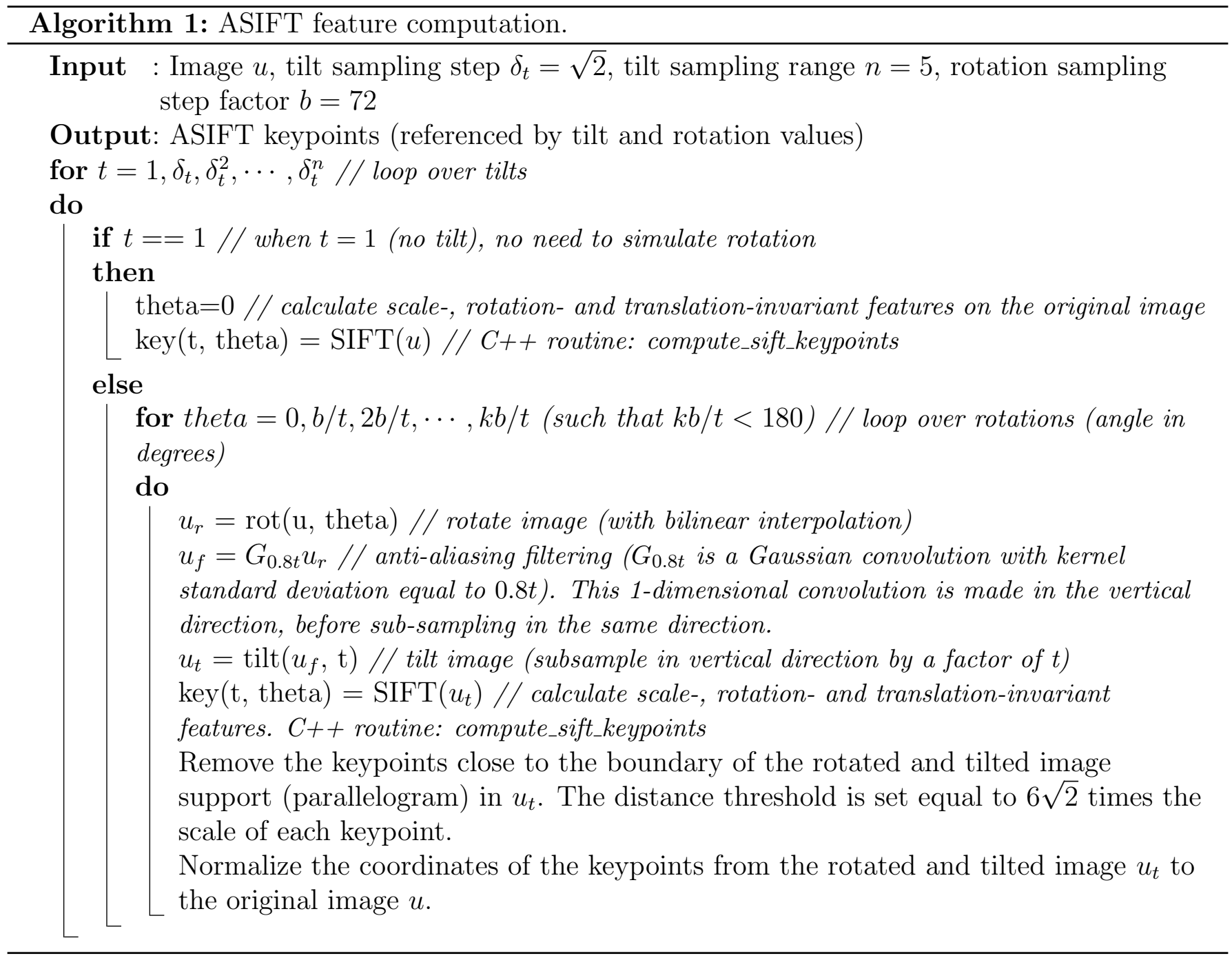

\subsection{ASIFT Feature Comparison}

Algorithm 2. Implemented in the $\mathrm{C}++$ source file compute_asift_matches.cpp. 


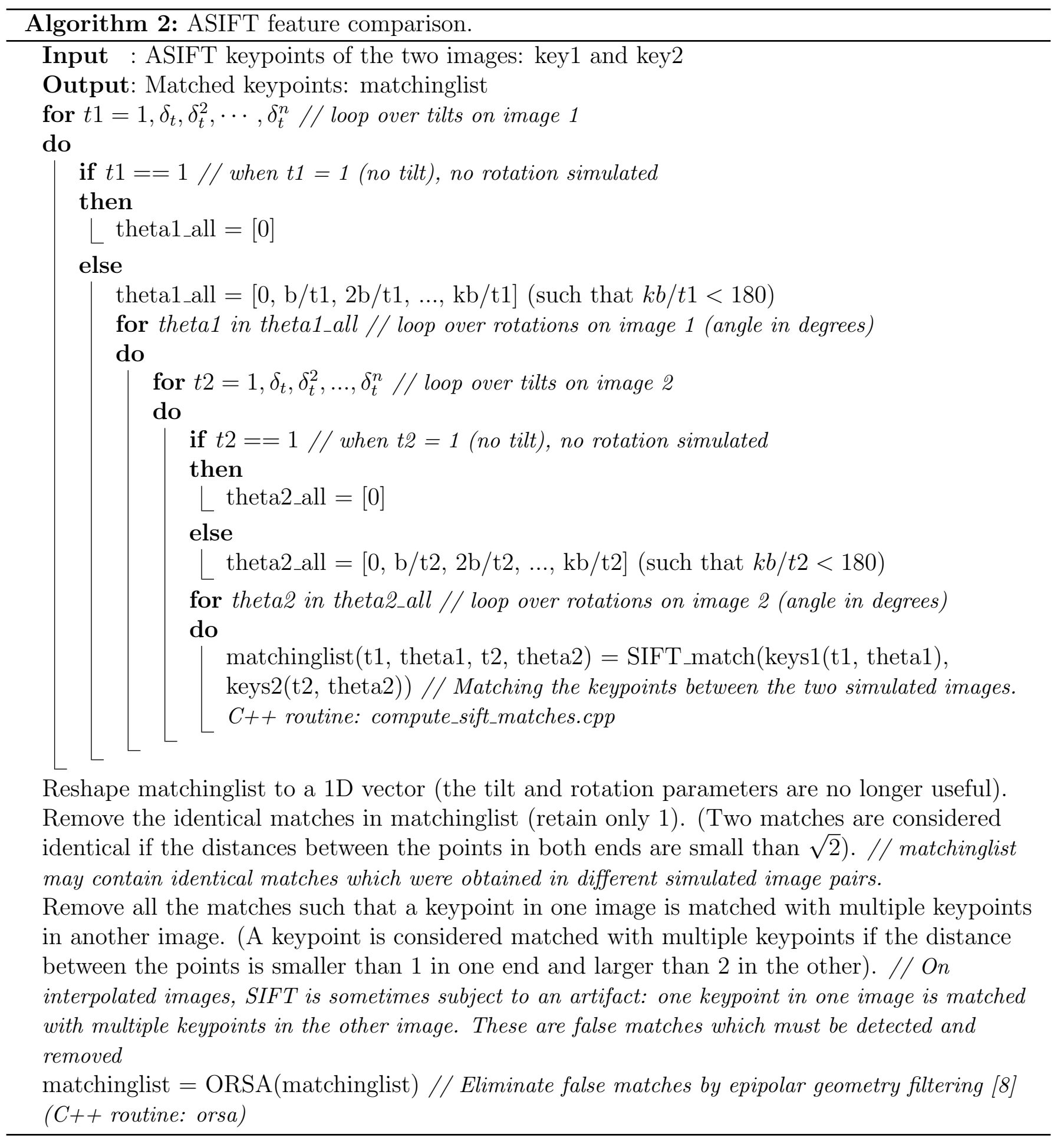

\section{Examples}

- Video tracking: Figure 7.

- Planar objects: figures 8, 9, 10, 11, 12, 13, 14, 15, 16, 17, 18.

- Monuments and constructions: figures 19, 20 and 21.

- 3D objects: figures 22 and 23.

- Complex scenes: figures 24 and 25. 
- Object deformation: figures 26, 27, 28, 29 and 30.

- Failure cases: figures 31 and 32 .

The matches are connected by white segments.
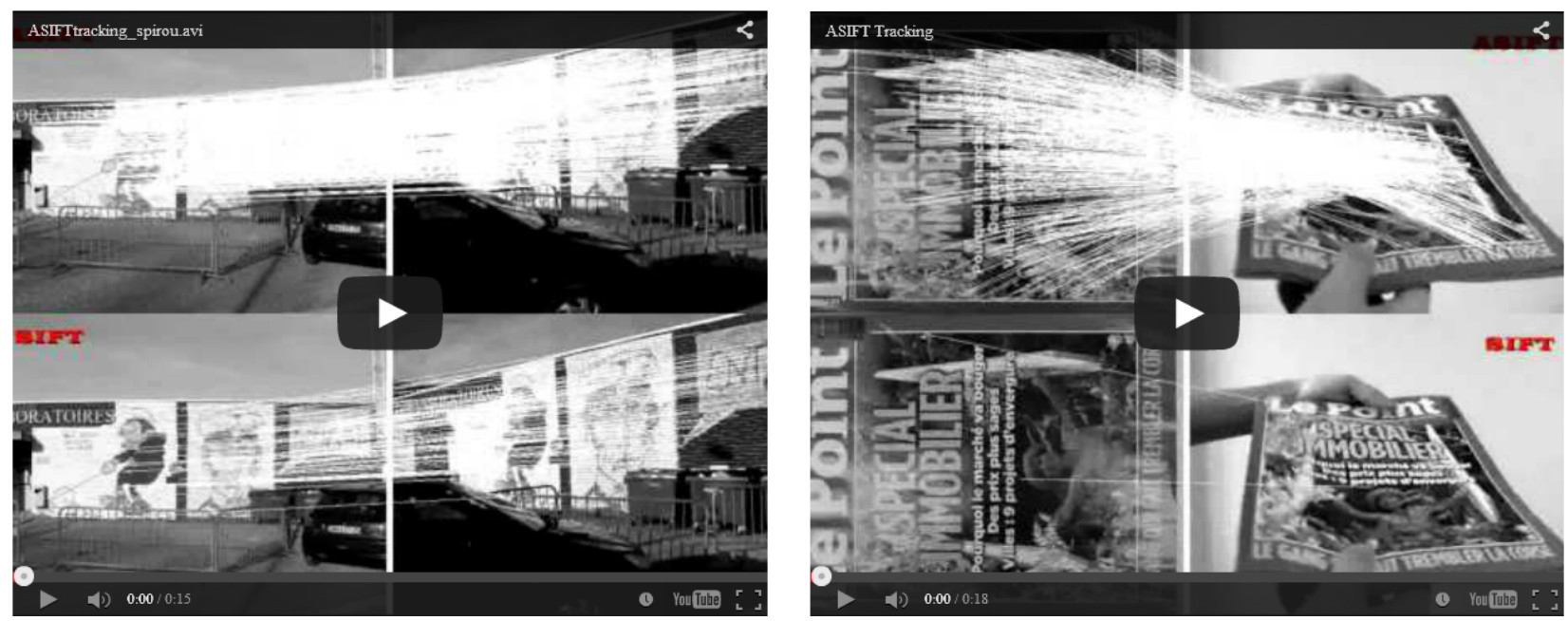

Figure 7: Left, facade. Right, magazine. ASIFT is compared with SIFT on video tracking. In each video, ASIFT and SIFT tracking are shown respectively on the top and bottom. The video files can be accessed by clicking the links below ${ }^{2}$.

\section{ASIFT: 202 matches}
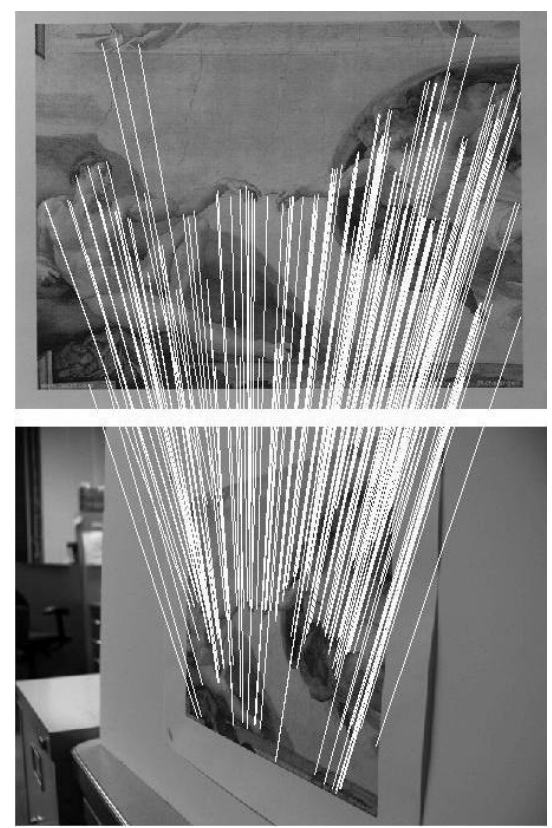

SIFT: 15 matches
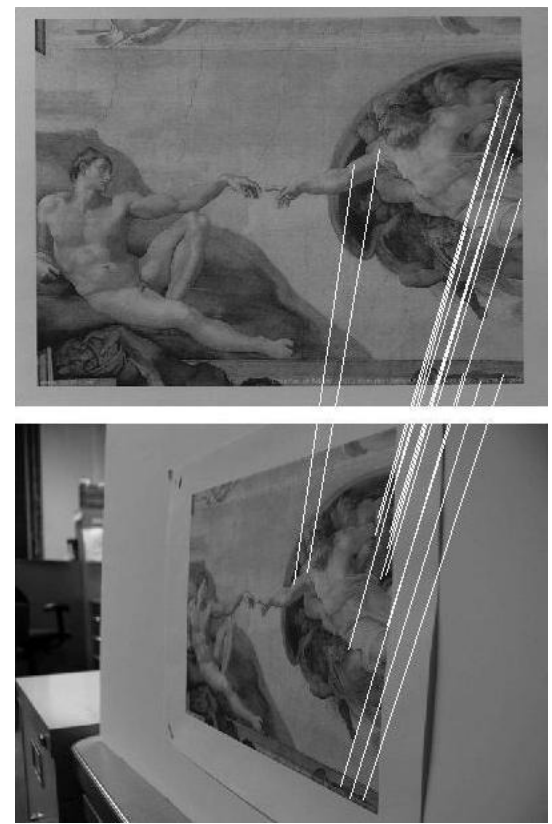

MSER: 5 matches
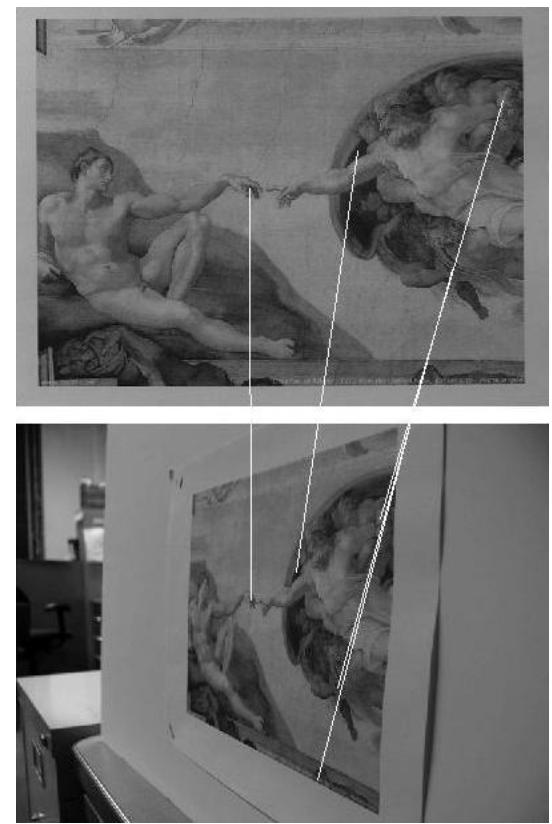

Figure 8: Adam, short distance $($ zoom $\times 1)$ at frontal view and at 75 degree angle, absolute tilt $t=4$ (middle), $<4$ (left), $>4$ (right). Not shown: Harris-Affine (3 matches), Hessian-Affine (1 match).

\footnotetext{
${ }^{2}$ Facade: Youtube video: http://www . youtube.com/watch?v=iY6d5pBdRC8

High-resolution video: http://www.ipol.im/data/algo/my_affine_sift/ASIFTtracking_spirou.avi

Magazine: Youtube video: http://www. youtube.com/watch?v=RU6jN-UOTwM

High-resolution video: http://www.ipol.im/data/algo/my_affine_sift/ASIFTtracking_lepoint.avi
} 

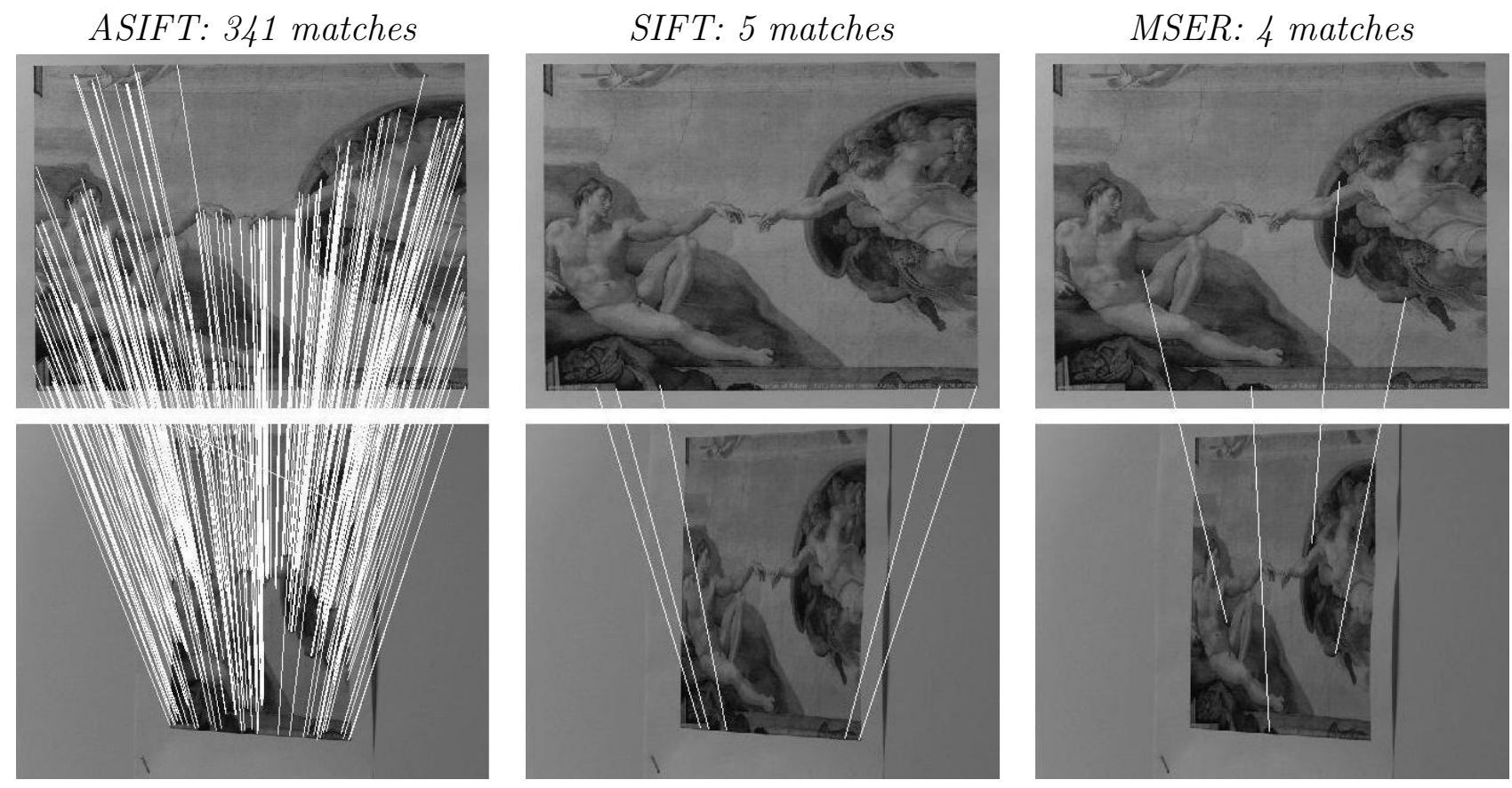

Figure 9: Adam, short distance $($ zoom $\times 10)$ at frontal view and at 65 degree angle, absolute tilt $t=2.4$ Not shown: Harris-Affine (3 matches) Hessian-Affine (0 match).
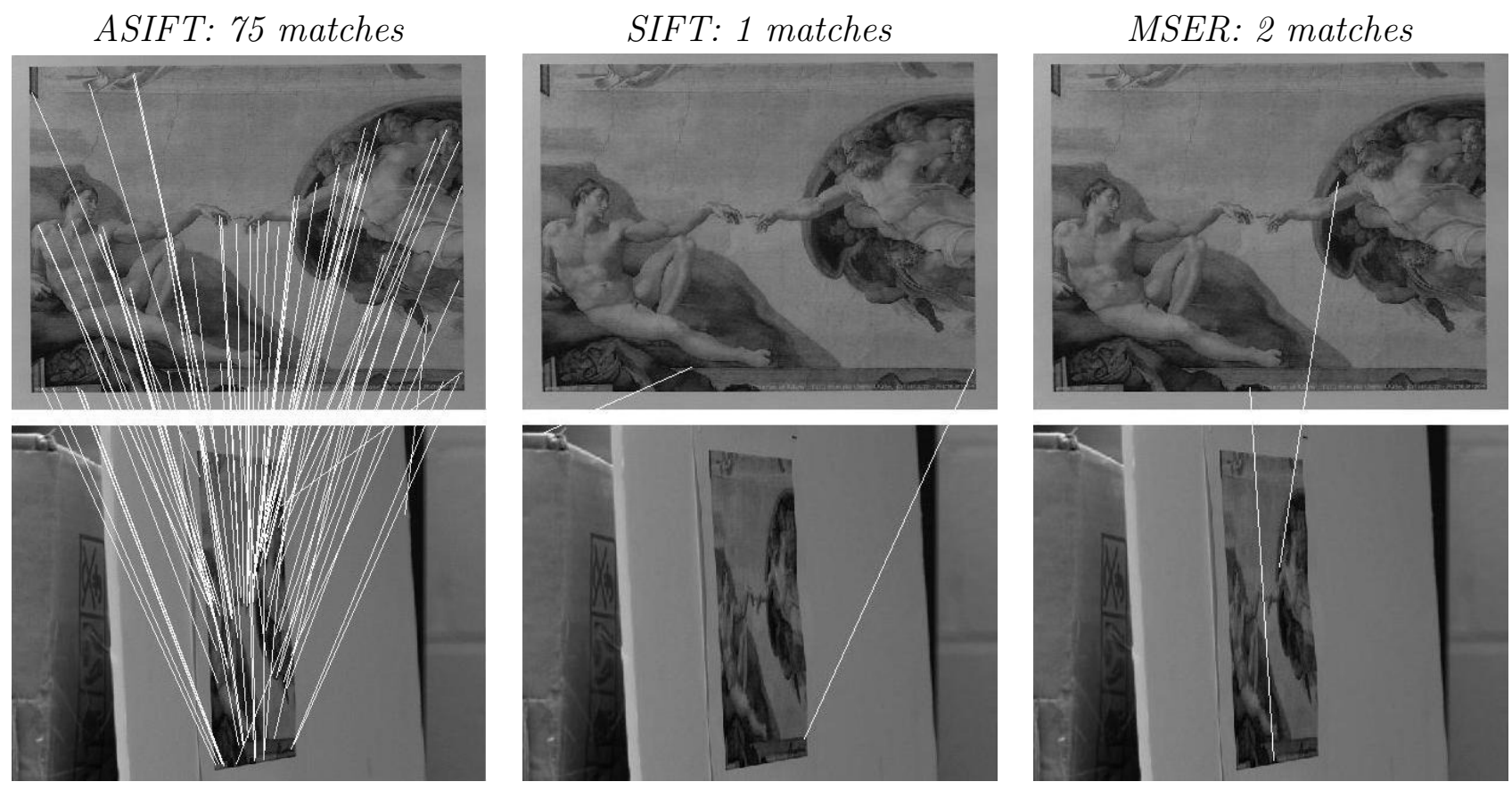

Figure 10: Adam, short distance $($ zoom $\times 10)$ at frontal view and at 80 degree angle, absolute tilt $t=5.8$. Not shown: Harris-Affine (1 match) Hessian-Affine (0 match). 

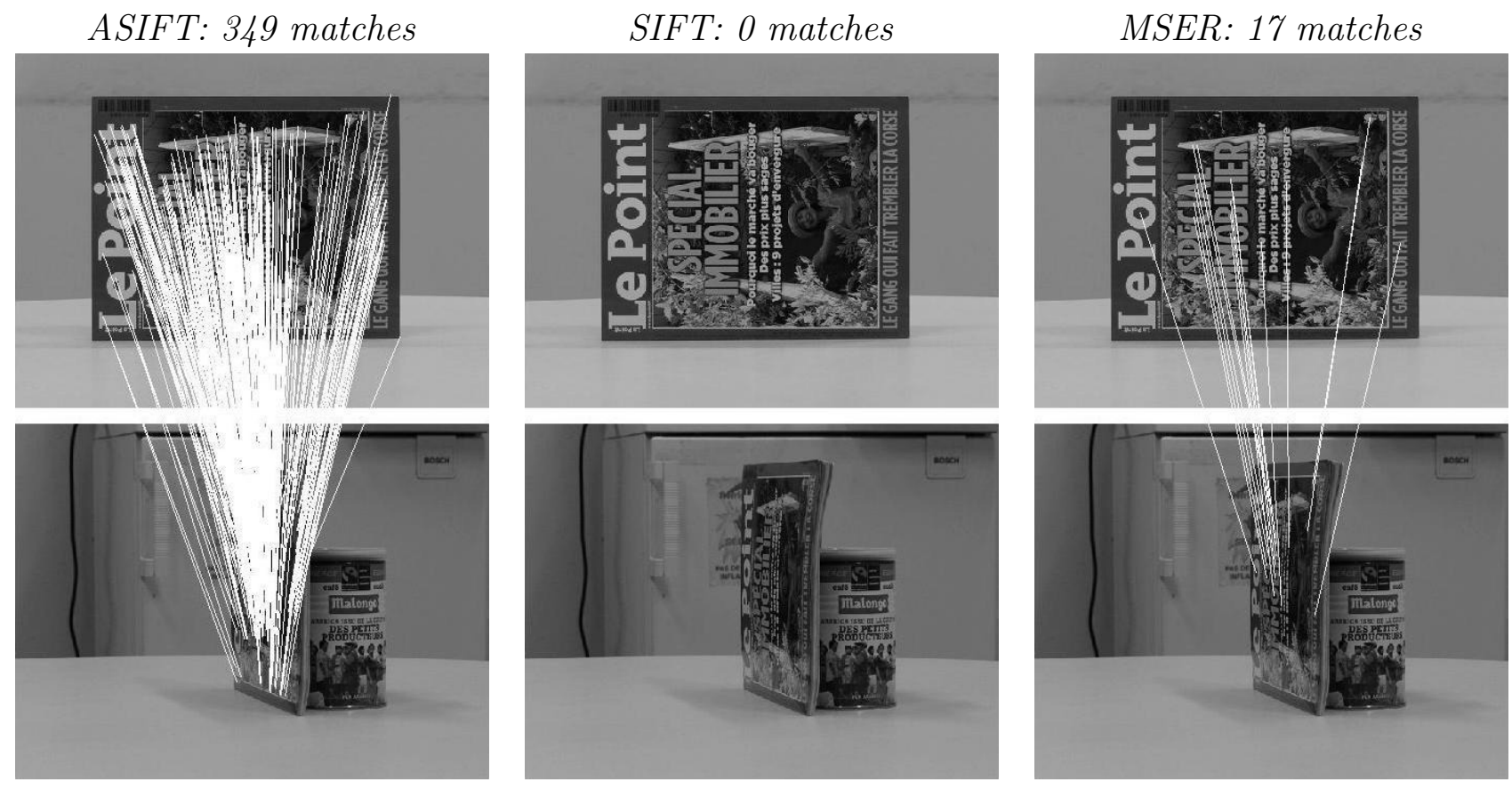

Figure 11: Magazine, middle distance $($ zoom $\times 4)$ at frontal view and at 80 degree angle, absolute tilt $t=5.8$. Not shown: Harris-Affine (0 match), Hessian-Affine (0 match).
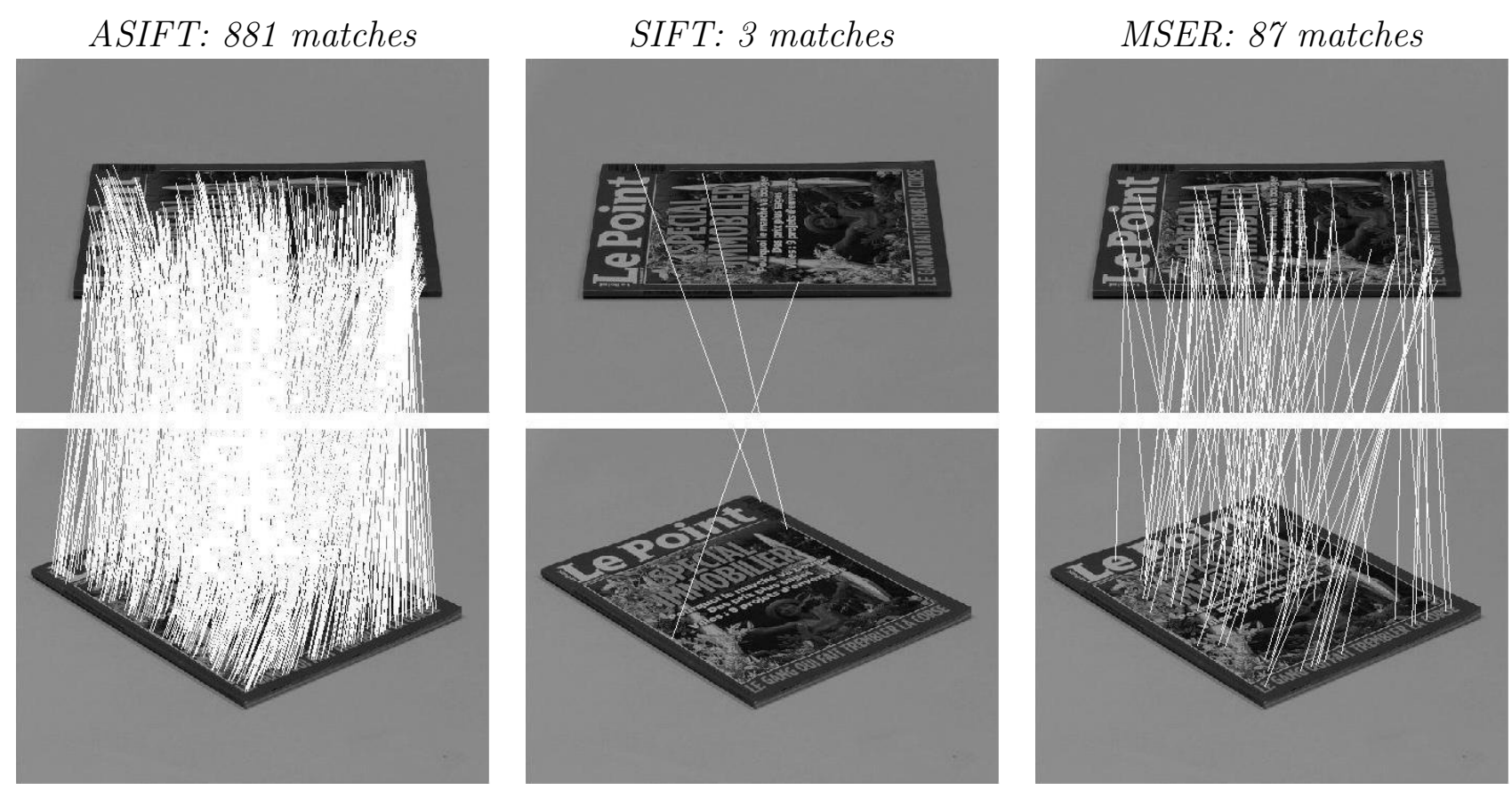

Figure 12: Magazine, absolute tilt $t 1=t 2=2$, with longitude angles phi1 $=0$ deg, phi2 $=50$ deg, transition tilt $t=3$. Not shown: Harris-Affine (0 match), Hessian-Affine (0 match). 

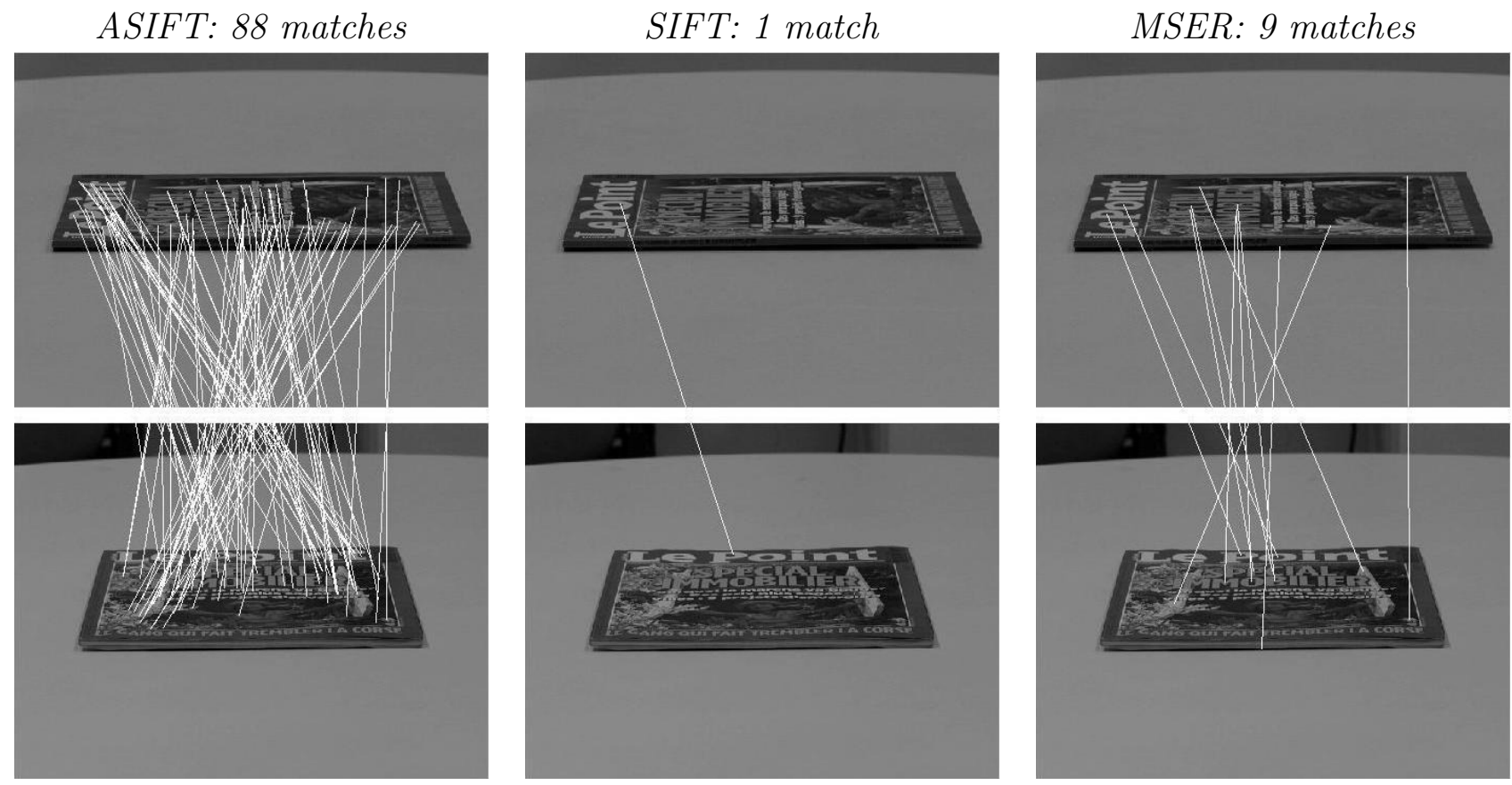

Figure 13: Magazine, absolute tilt $t 1=t 2=4$, with longitude angles phi1 $=0$ deg, phi2 $=90$ deg, transition tilt $t=16$. Not shown: Harris-Affine (0 match), Hessian-Affine (0 match). 
ASIFT: 68 matches
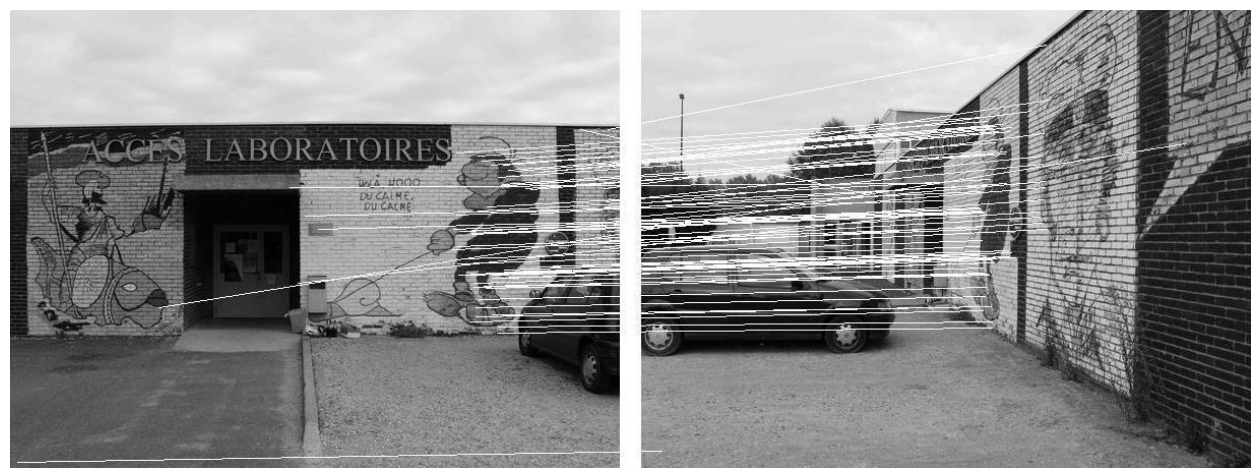

Hessian-Affine: 1 match
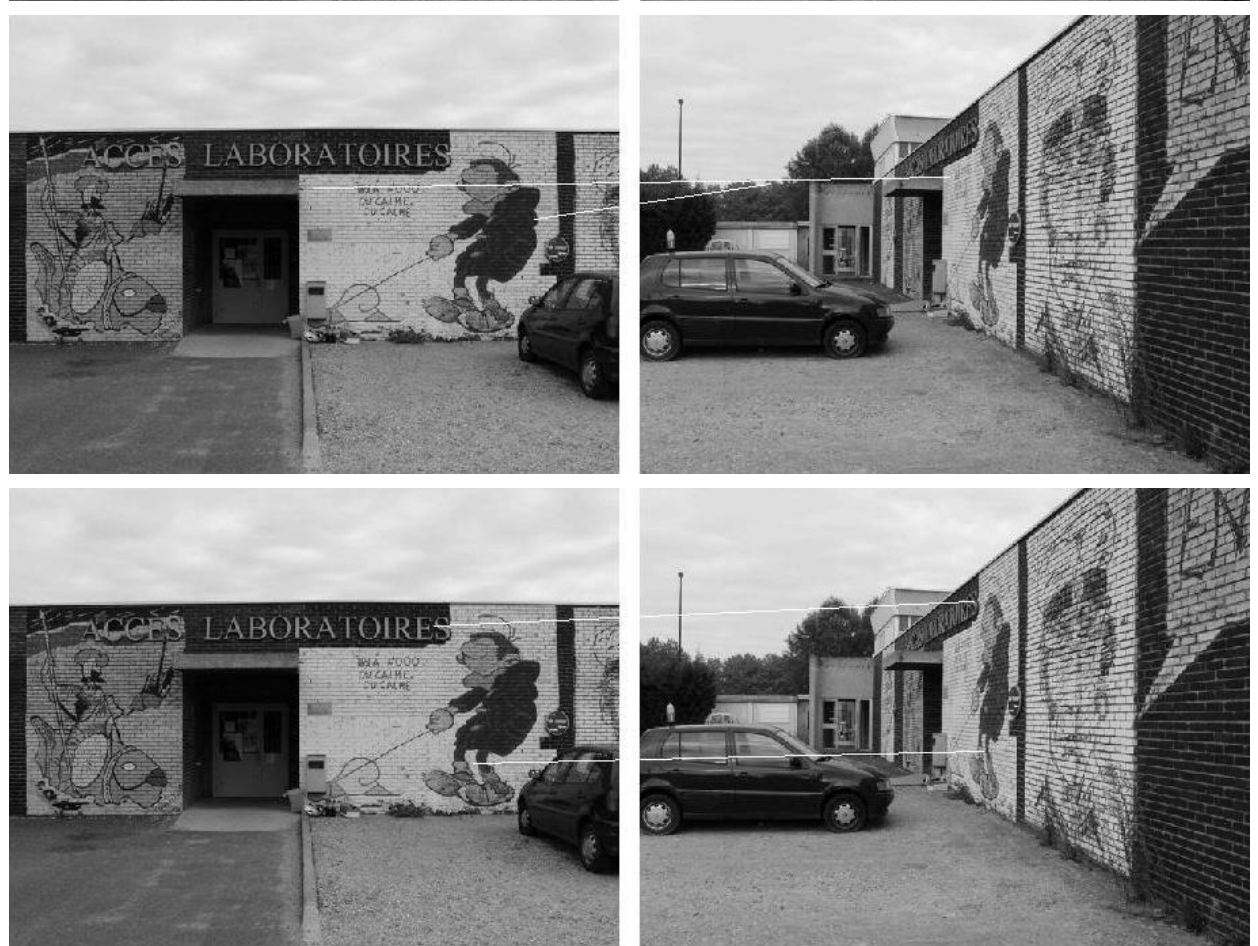

MSER: 2 matches

Figure 14: Facade, frontal view and at 75 degree angle, absolute tilt $t=3.8$ Not shown: SIFT $(0$ match), Harris-Affine (1 match). 
ASIFT: 721 matches

Hessian-Affine: 3 matches
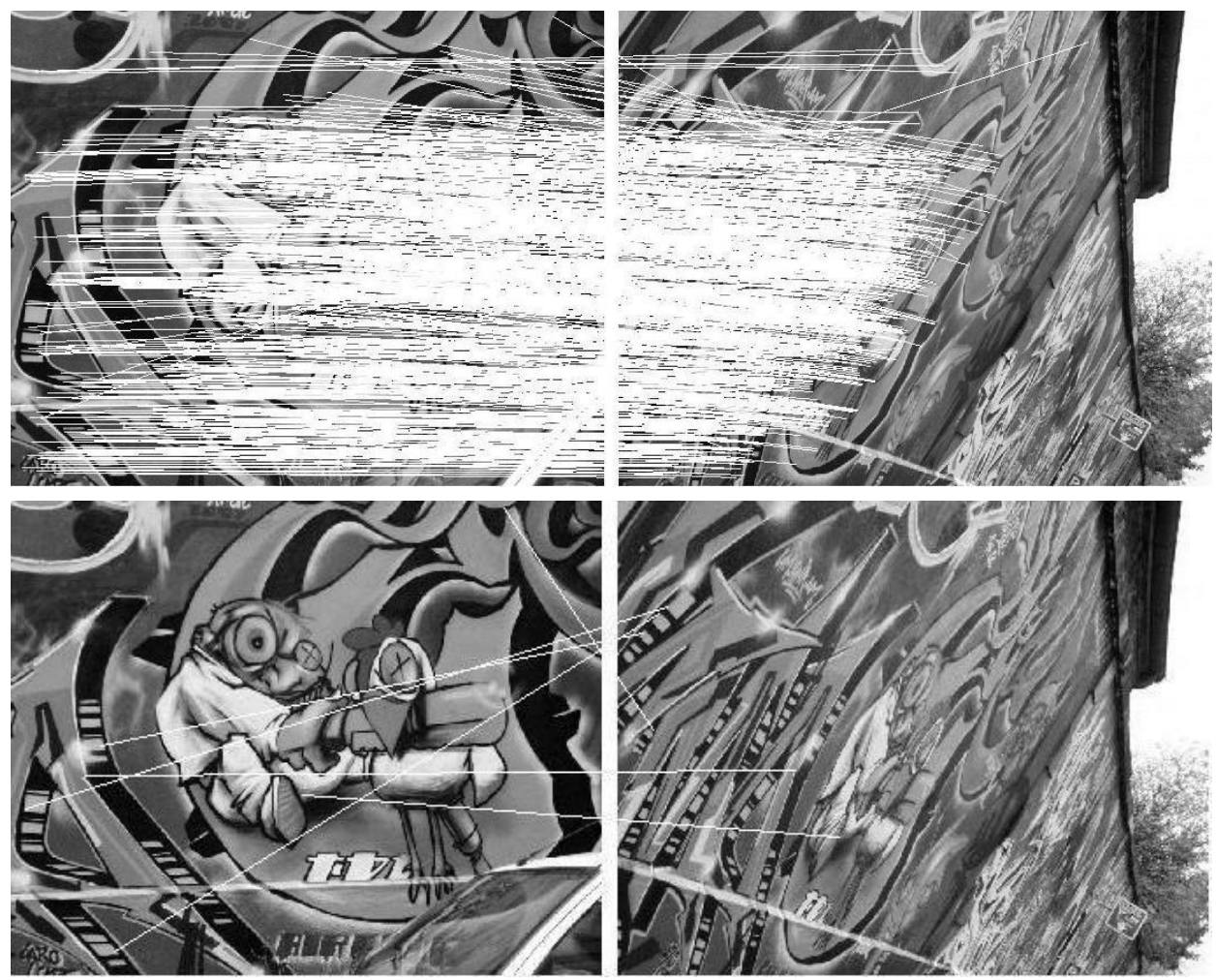

MSER: 70 matches
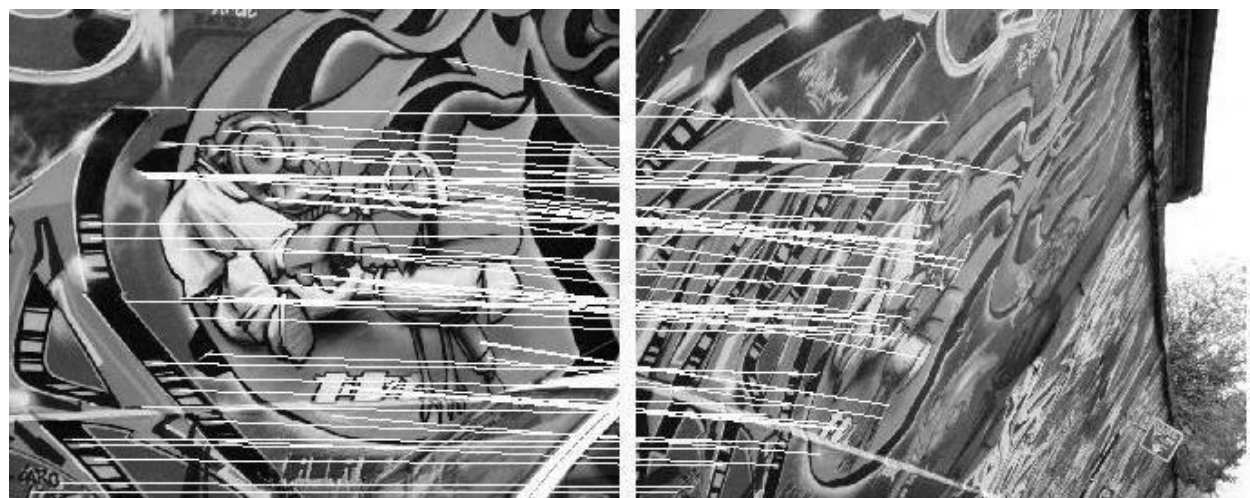

Figure 15: graffiti no.1 vs no.6, transition tilt $t \sim 3.2$. Not shown: SIFT (0 match), Hessian-Affine (1 match). 
ASIFT: 50 matches

SIFT: 0 matches
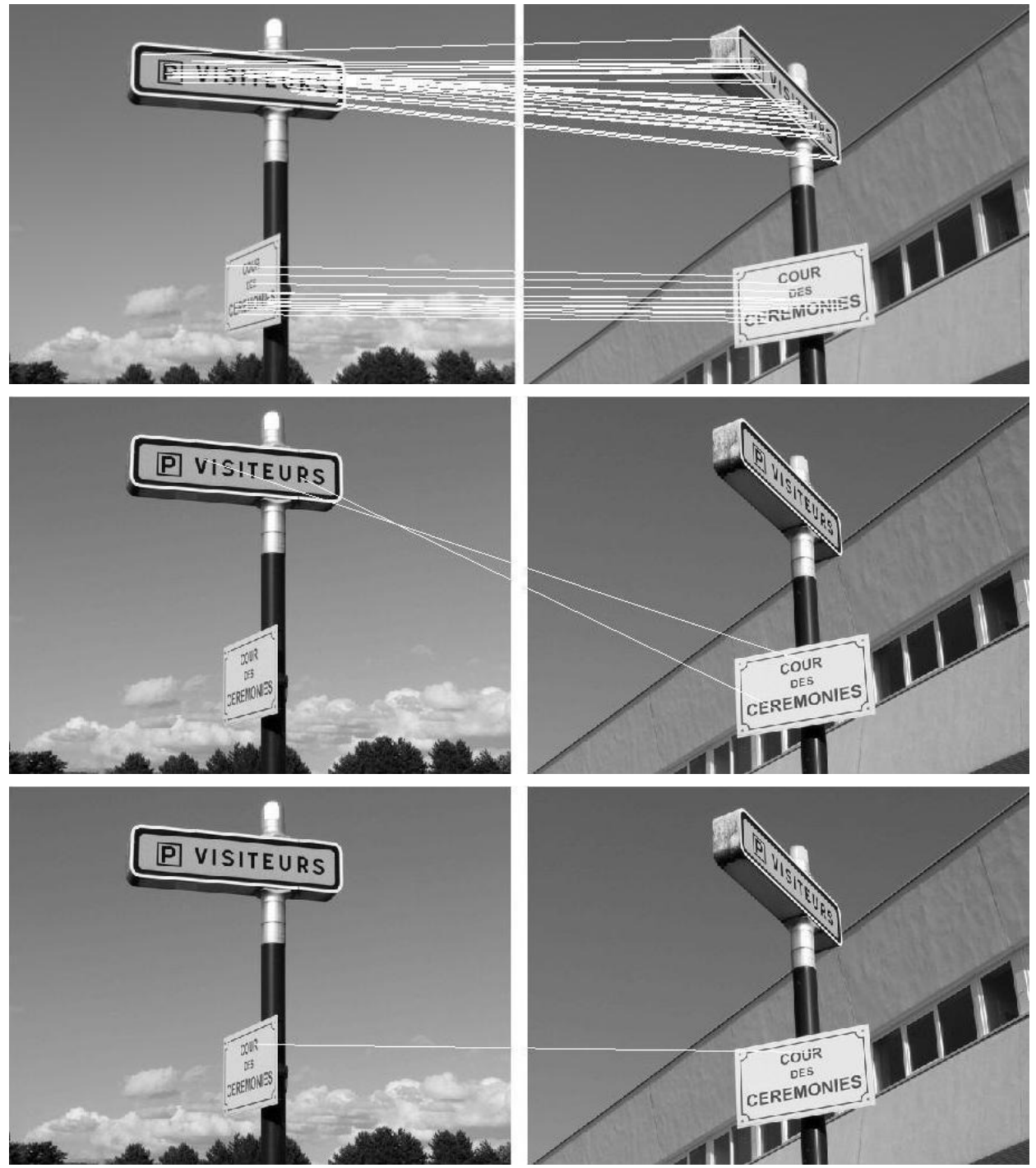

MSER: 1 match

Figure 16: Direction, transition tilt $t \sim 2.6$. Not shown: Harris-Affine (0 match), Hessian-Affine $(0$ match). 
ASIFT: 70 matches
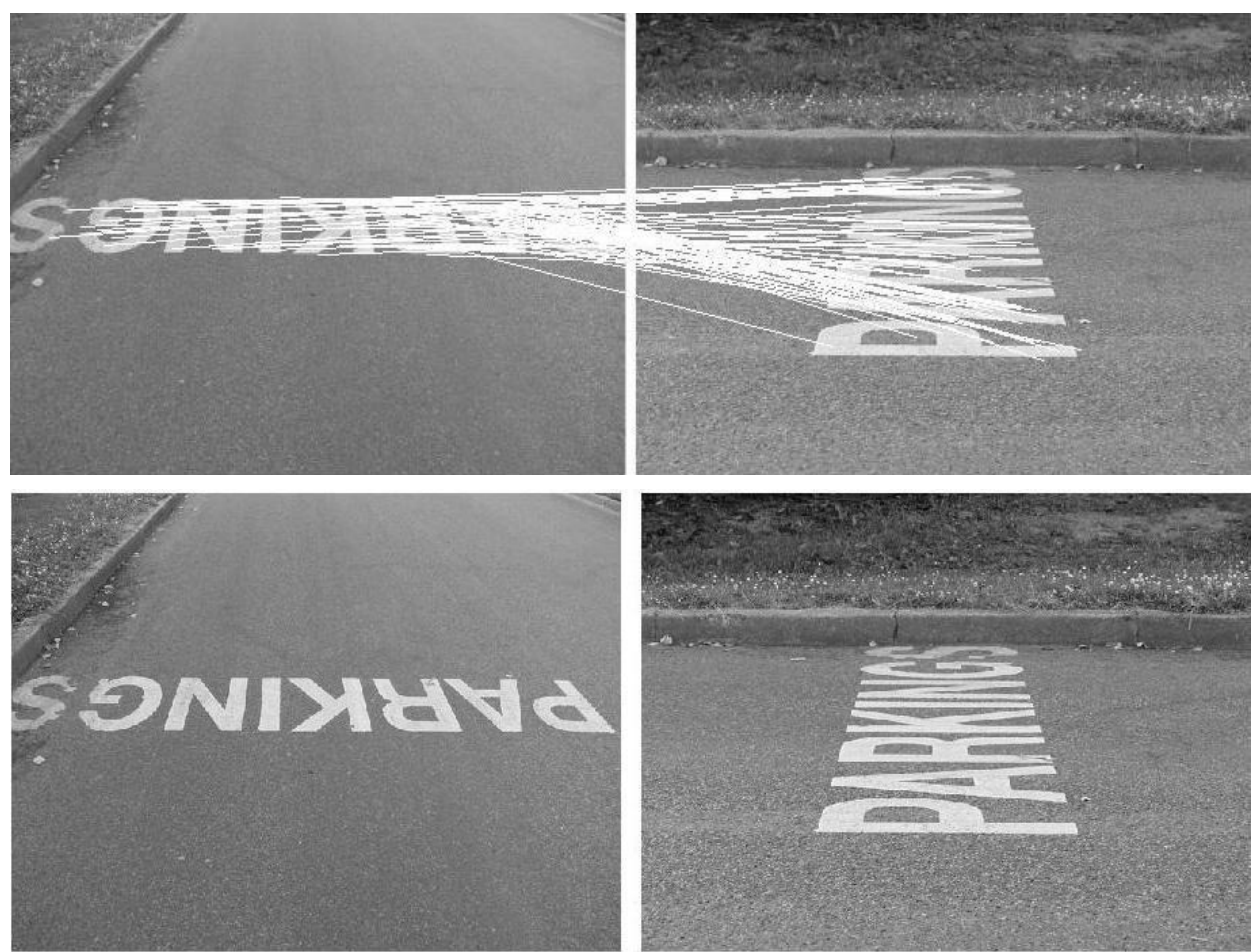

SIFT: 0 matches

MSER: 0 matches
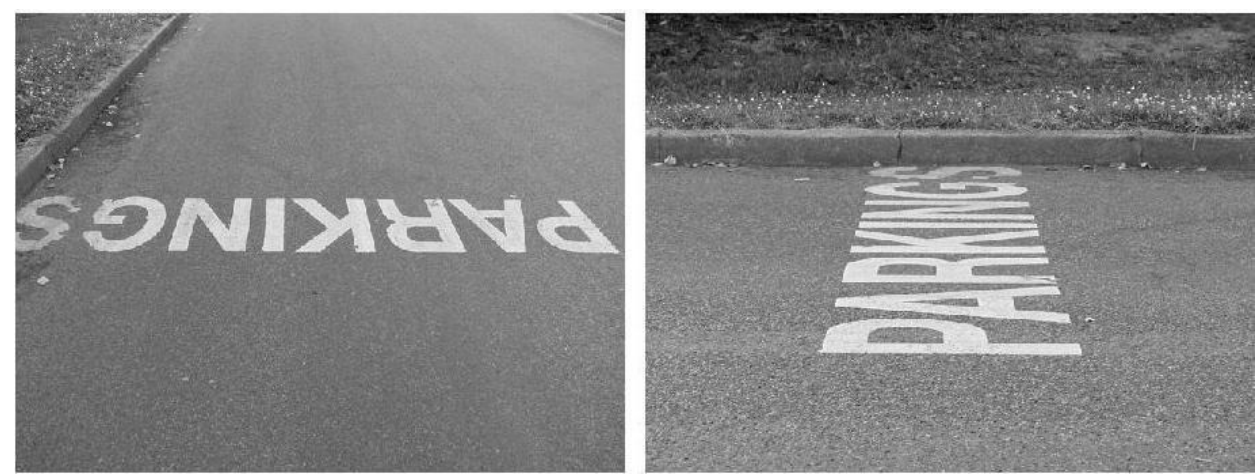

Figure 17: Parking, transition tilt $t \sim 15$. Not shown: Harris-Affine (0 match), Hessian-Affine $(0$ match). 

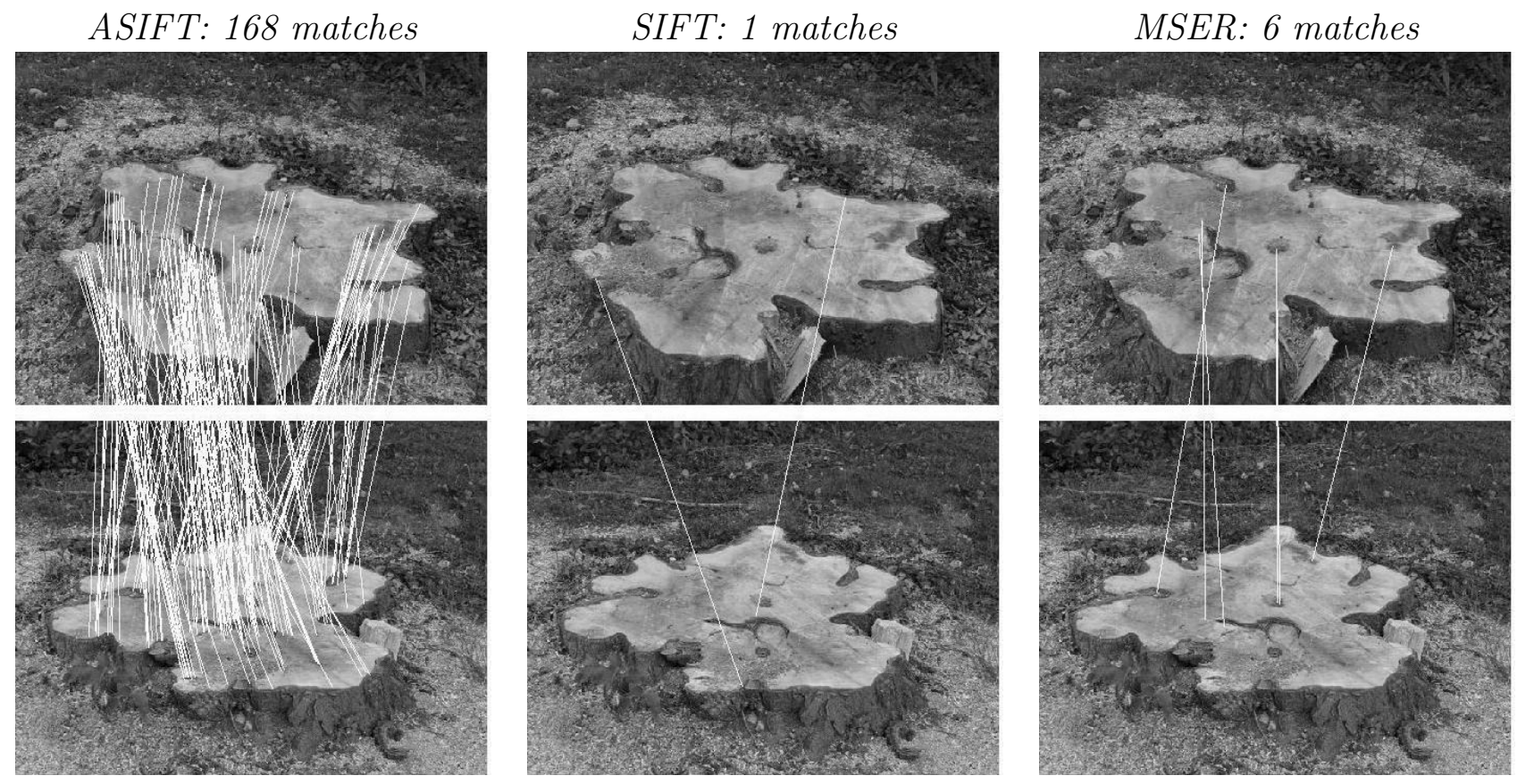

Figure 18: Stump, transition tilt $t \sim 2.6$. Not shown: Harris-Affine (2 matches), Hessian-Affine (1 match).
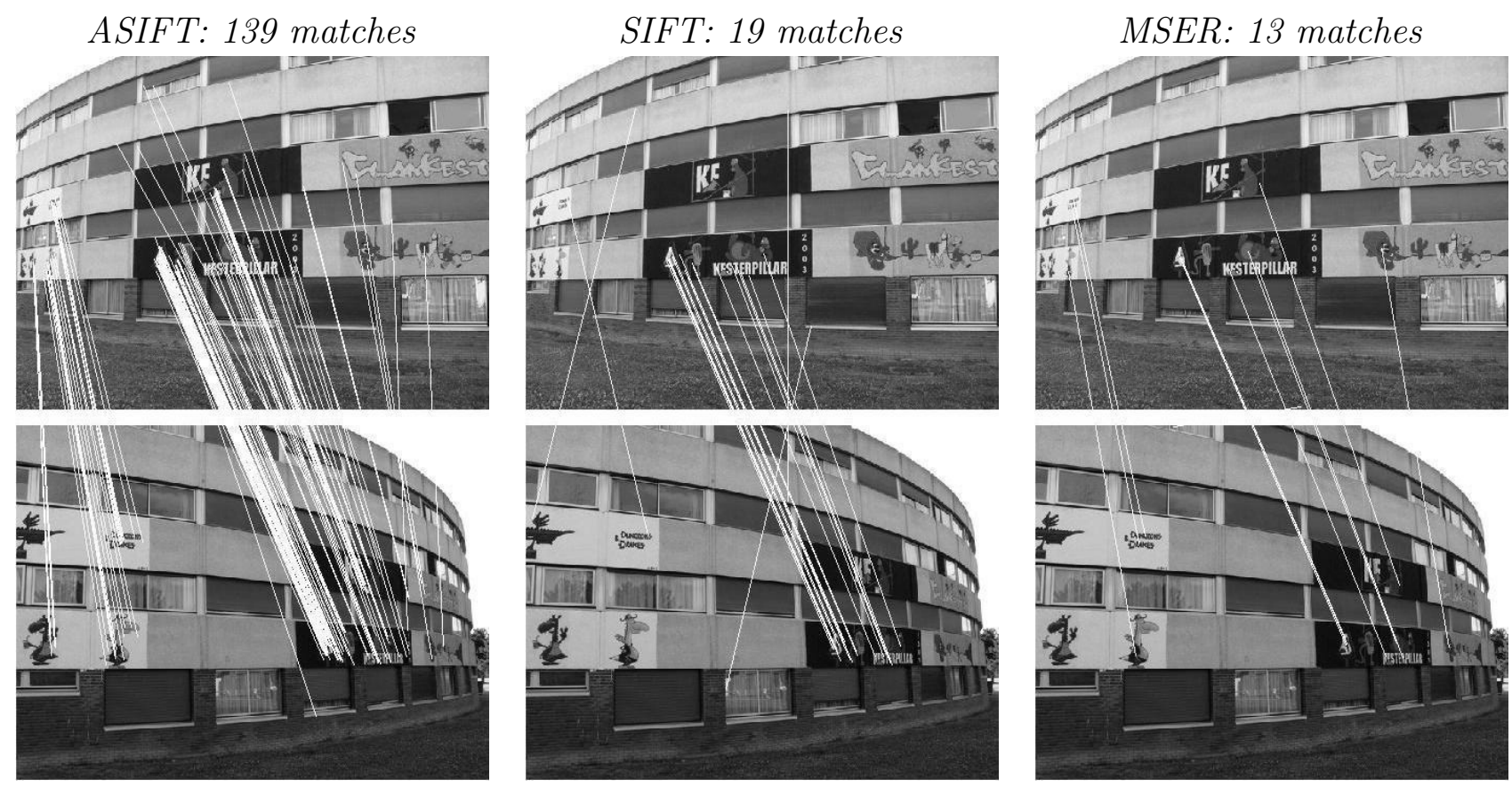

Figure 19: Round building, transition tilt $t \sim[1.8, \infty)$. Not shown: Harris-Affine (5 matches), Hessian-Affine (7 matches). 

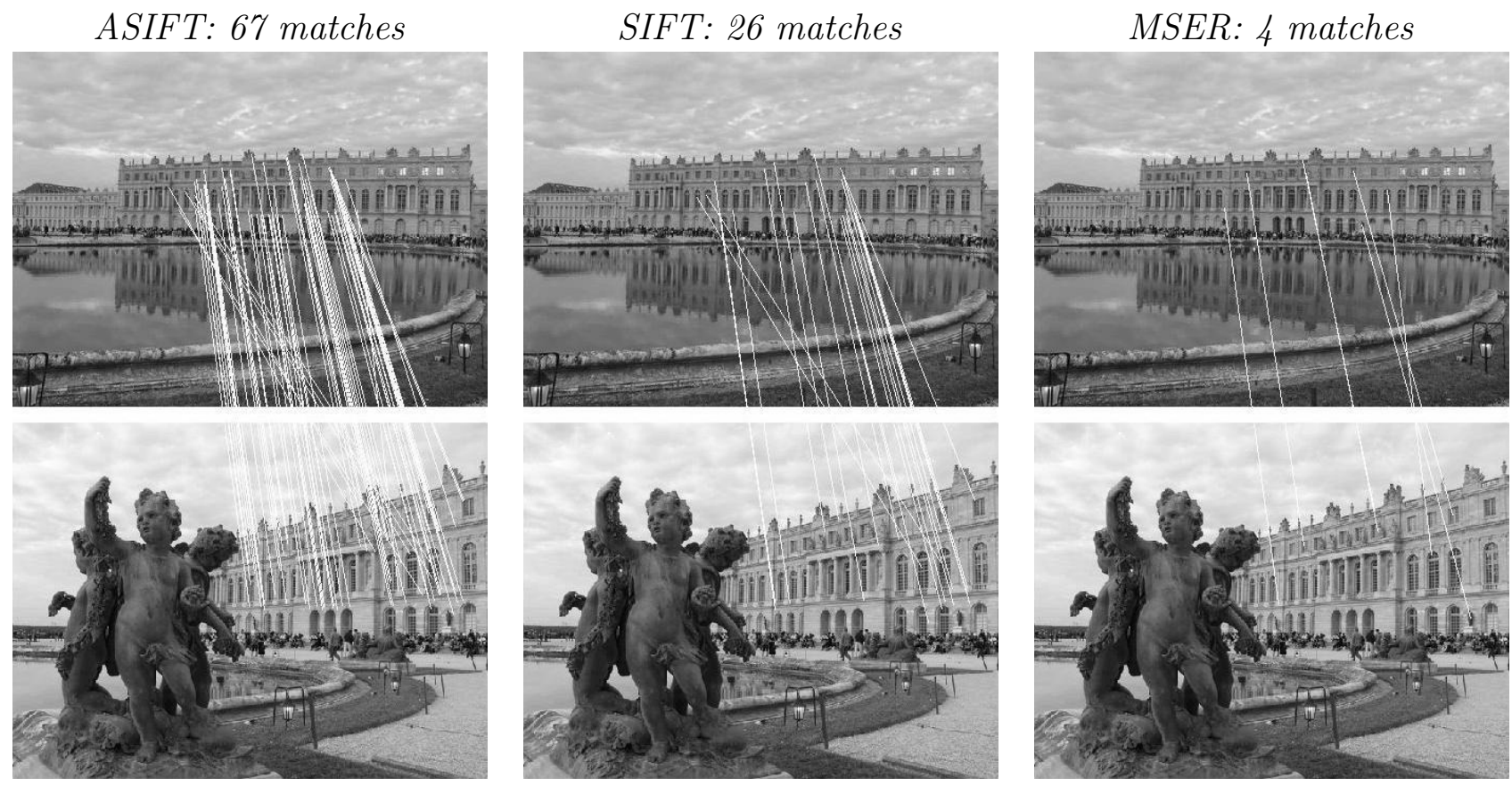

Figure 20: Palace of Versailles, transition tilt $t \sim 1.8$. Not shown: Harris-Affine (2 matches), Hessian-Affine (1 match).
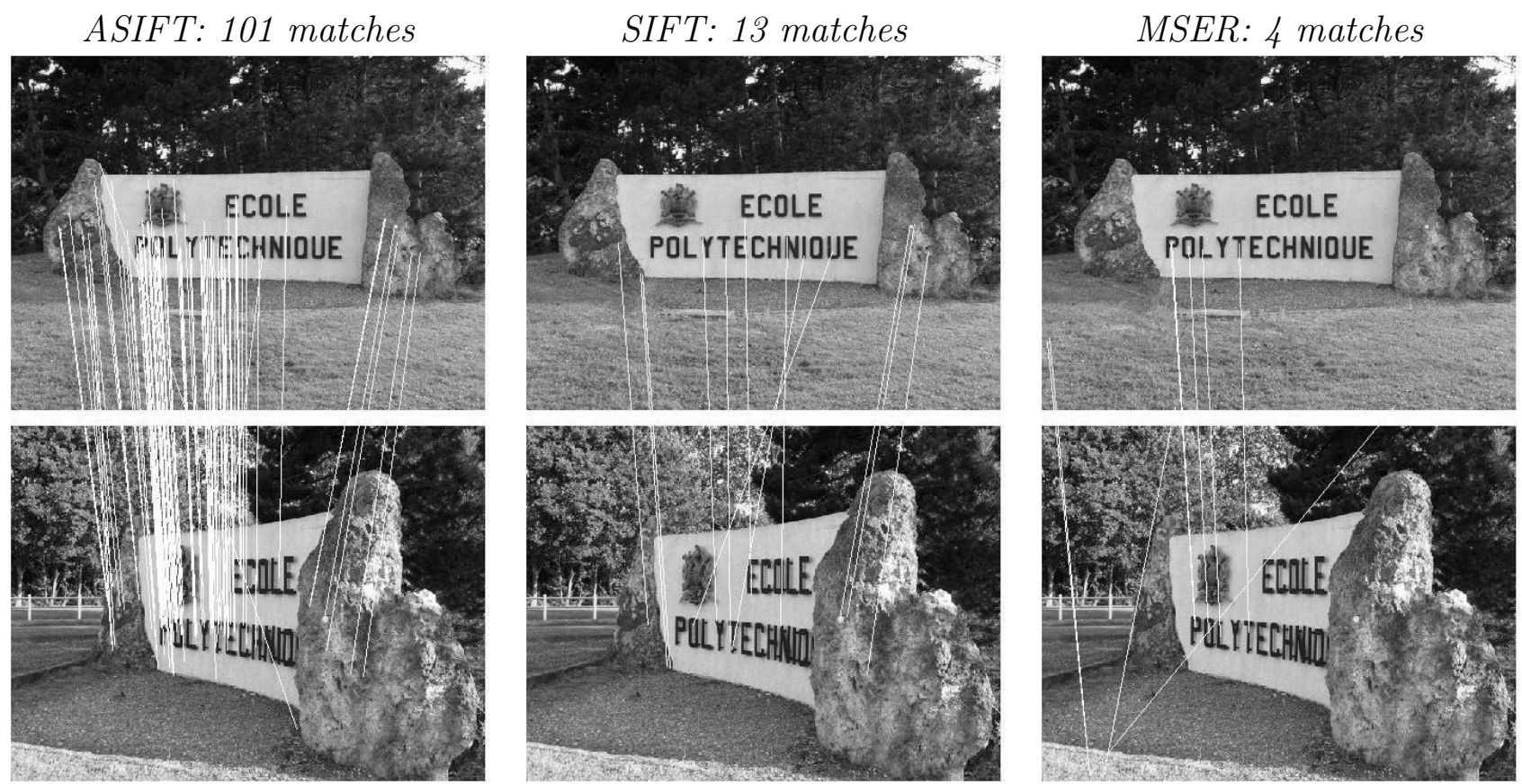

Figure 21: École Polytechnique, pictures at frontal view and at 65 degree angle, absolute tilt $t=2.4$. Not shown: Harris-Affine (2 matches), Hessian-Affine (2 matches). 

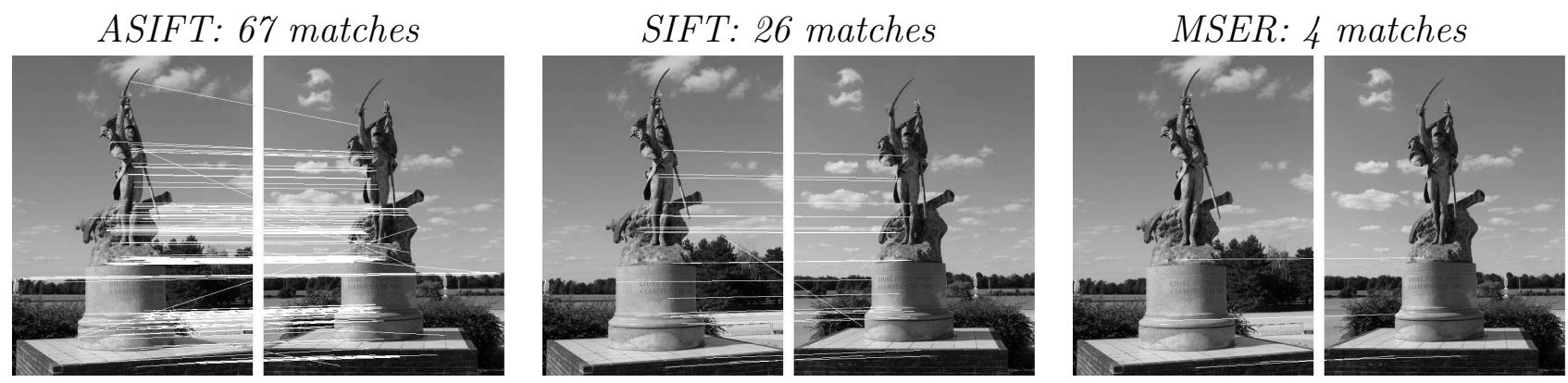

Figure 22: Statue, transition tilt $t \sim[1.6, \infty)$. Not shown: Harris-Affine (7 matches), Hessian-Affine (2 matches).

ASIFT: 287 matches

Harris-Affine: 6 matches
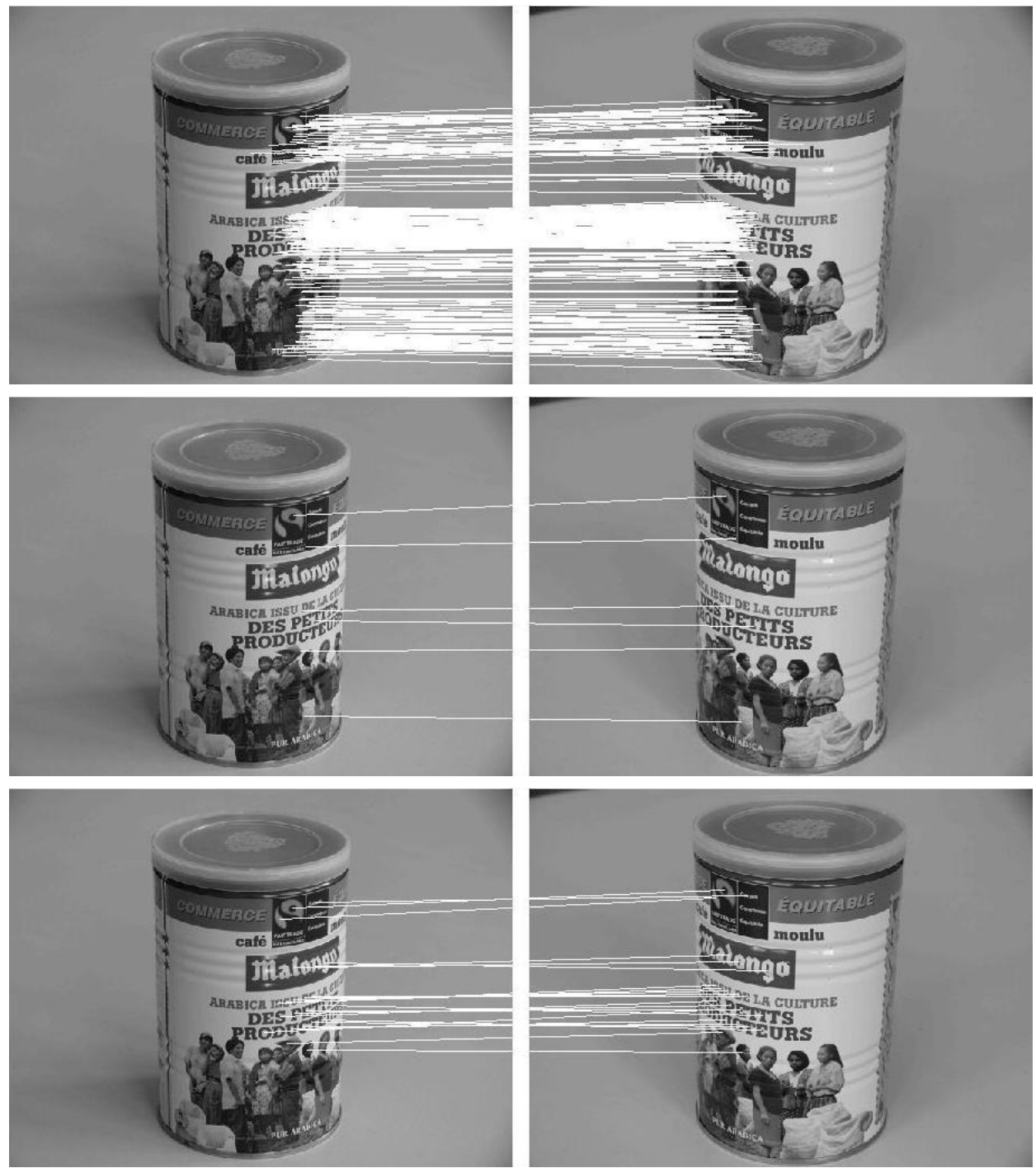

MSER: 22 matches

Figure 23: Can, transition tilt $t \sim[2.3, \infty)$. Not shown: SIFT (0 match), Hessian-Affine (3 matches). 
ASIFT: 88 matches
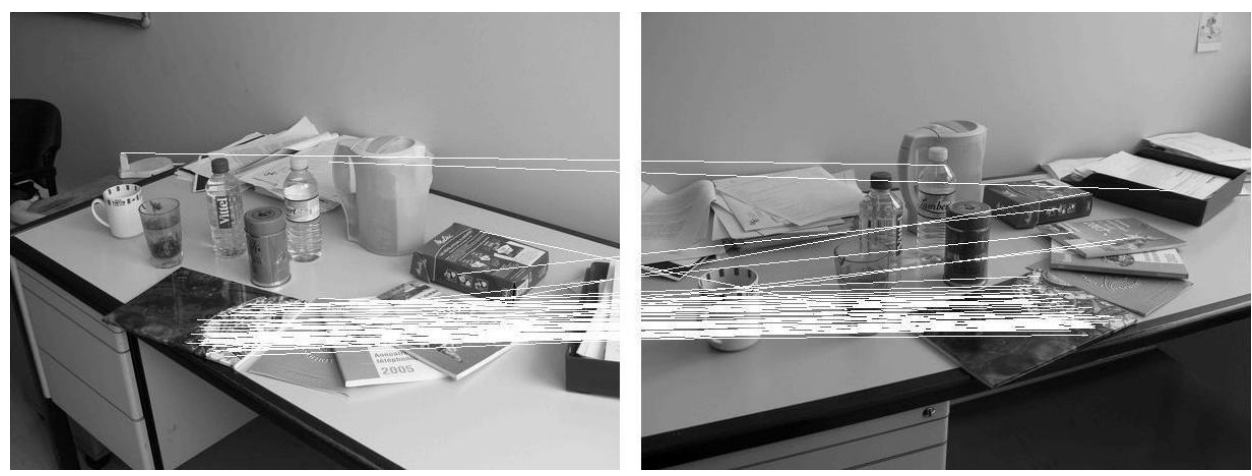

Hessian-Affine: 1 match
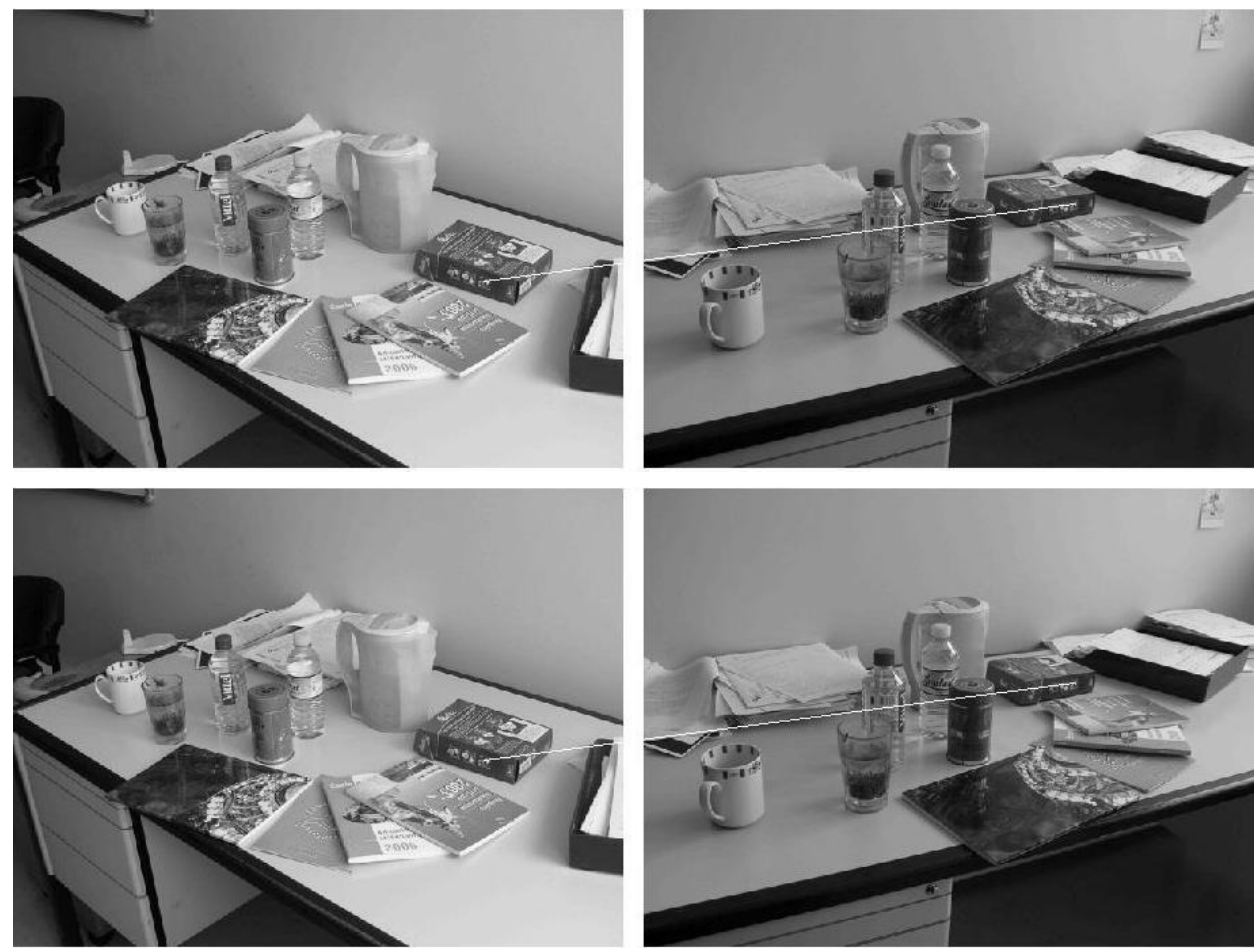

Figure 24: Office, transition tilt $t \sim 3$. Not shown: SIFT (0 match), Harris-Affine (0 match). 
ASIFT: 125 matches
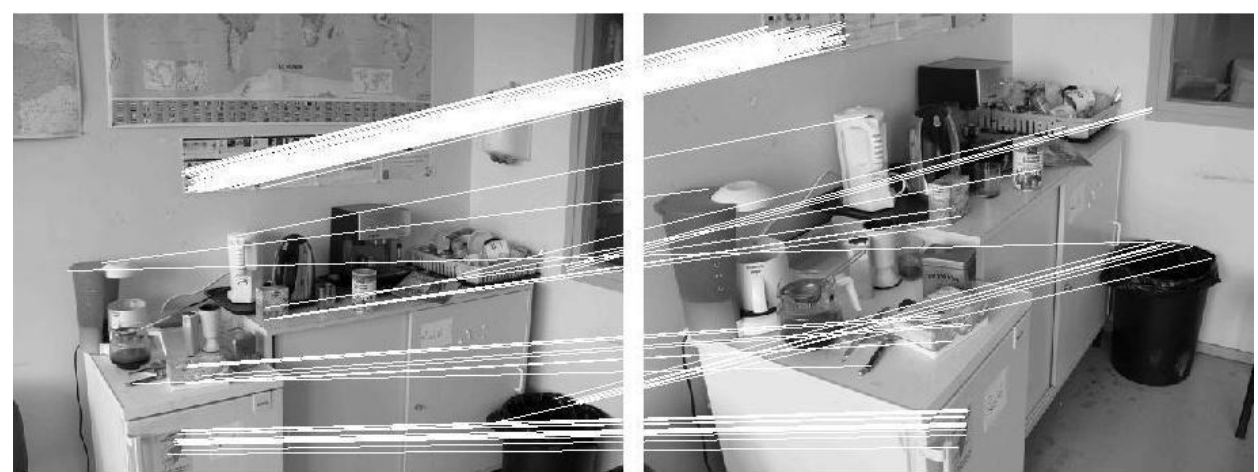

SIFT: 13 matches
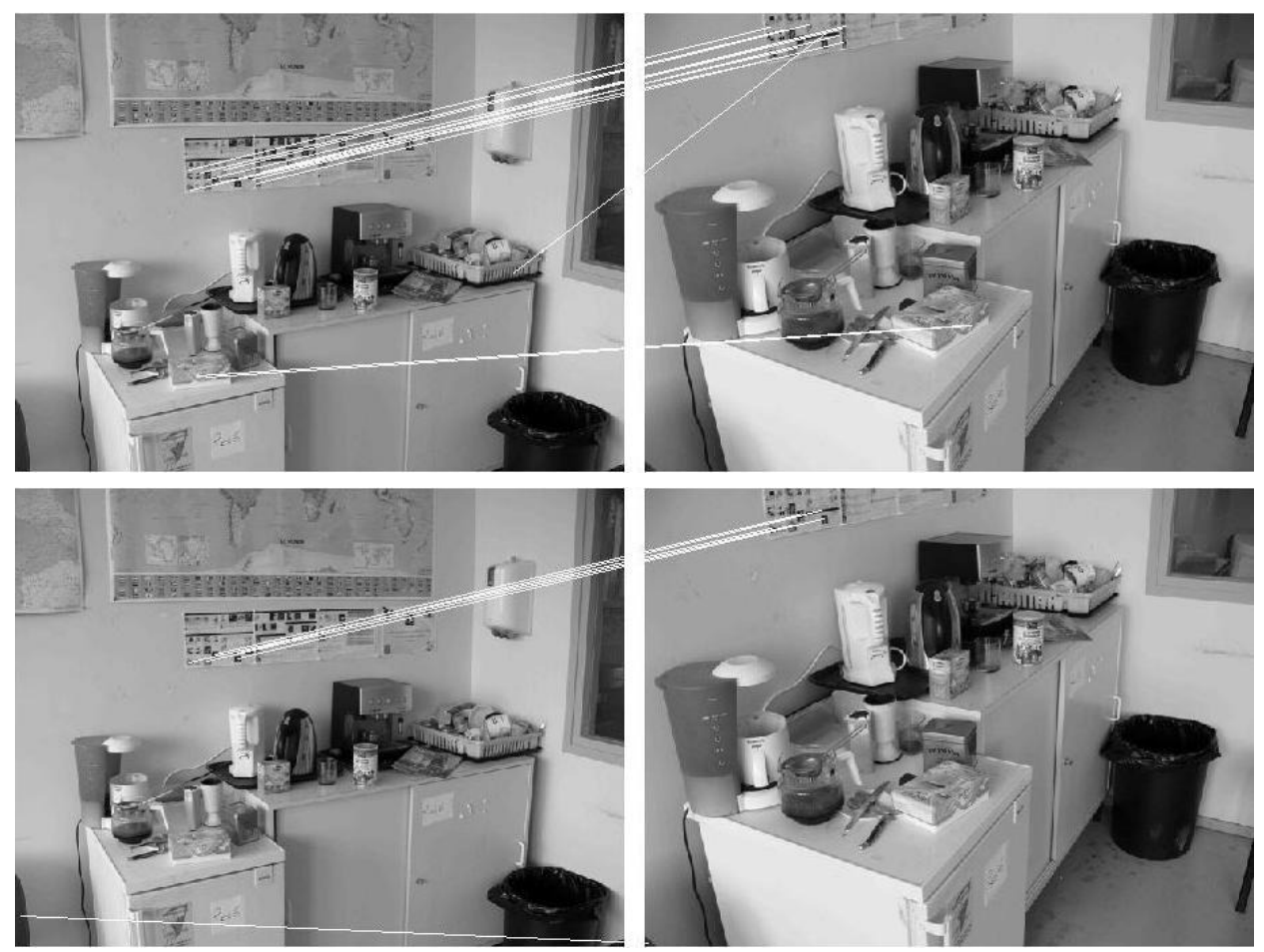

MSER: 5 matches

Figure 25: Coffee room transition tilt $t \sim[1.5,3.3]$. Not shown: Harris-Affine (0 match), HessianAffine (3 matches).
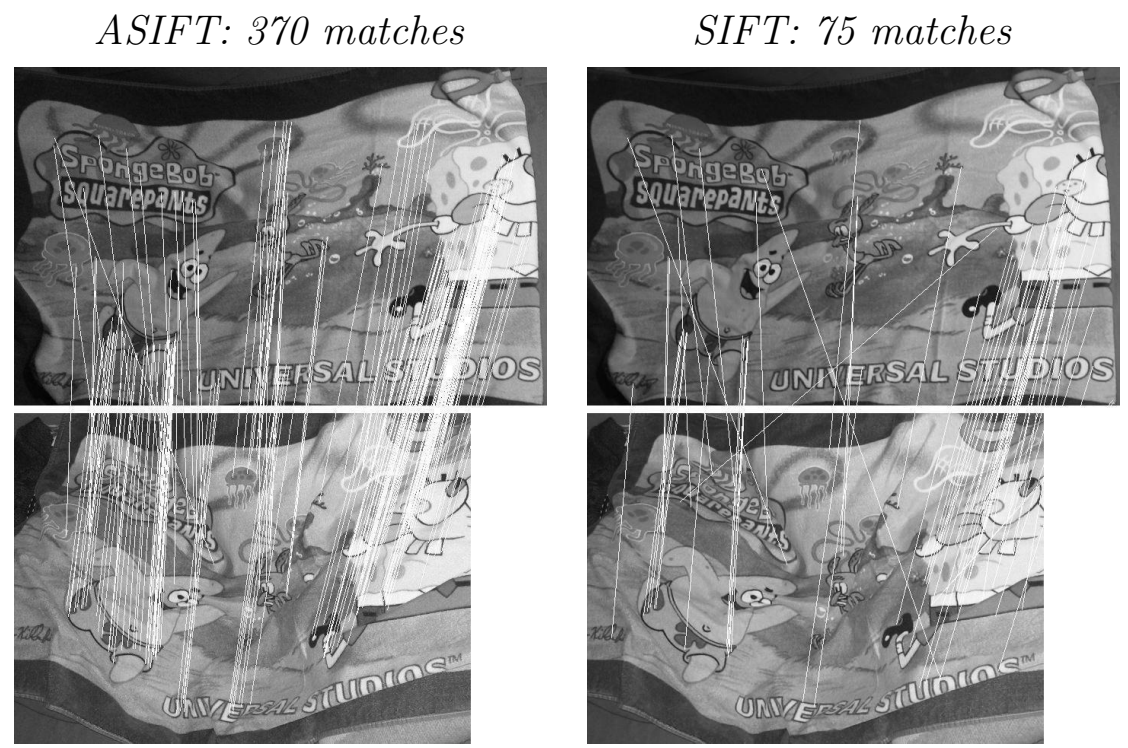

Harris-Affine: 8 matches
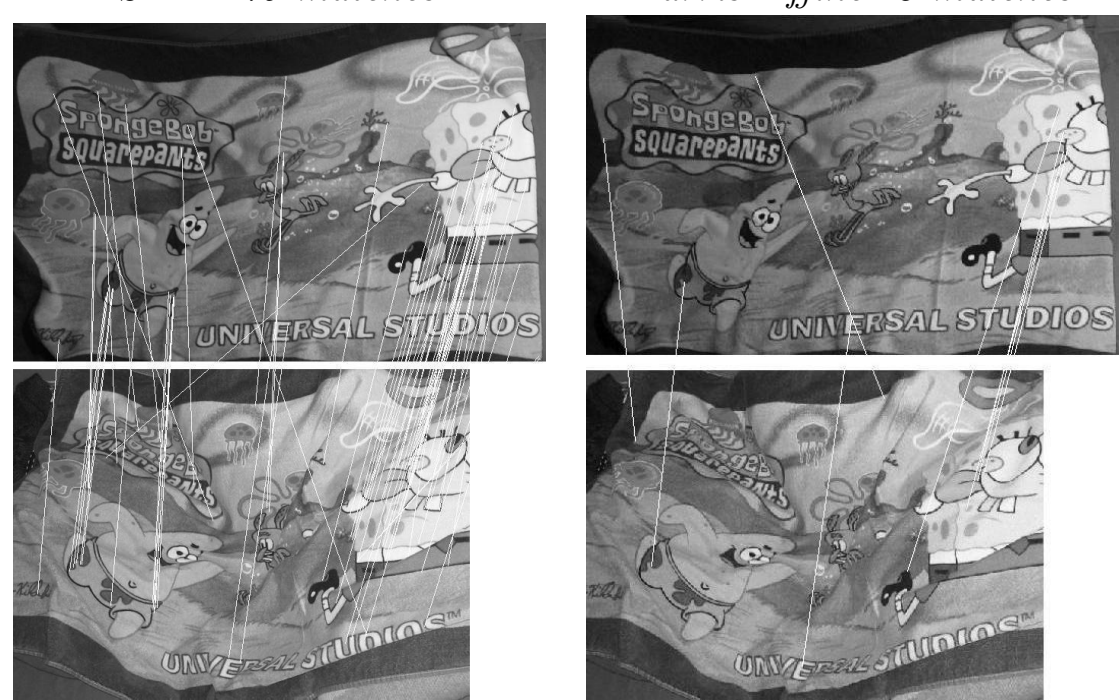

Figure 26: Sponge Bob. Not shown: Hessian-Affine (6 matches), MSER (4 matches). 
ASIFT: 528 matches

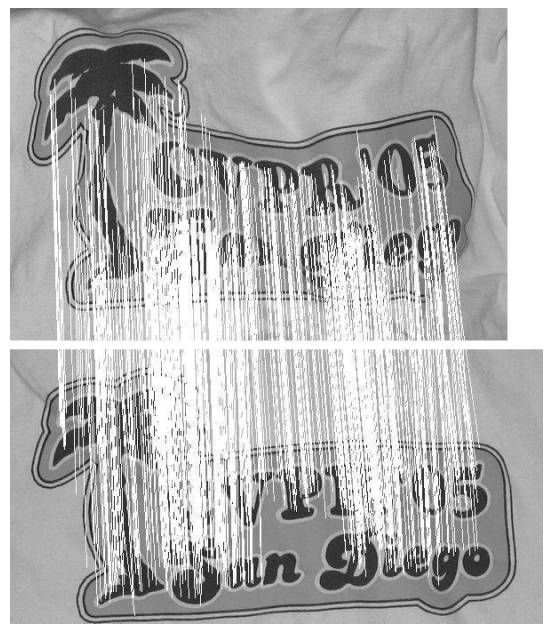

SIFT: 160 matches

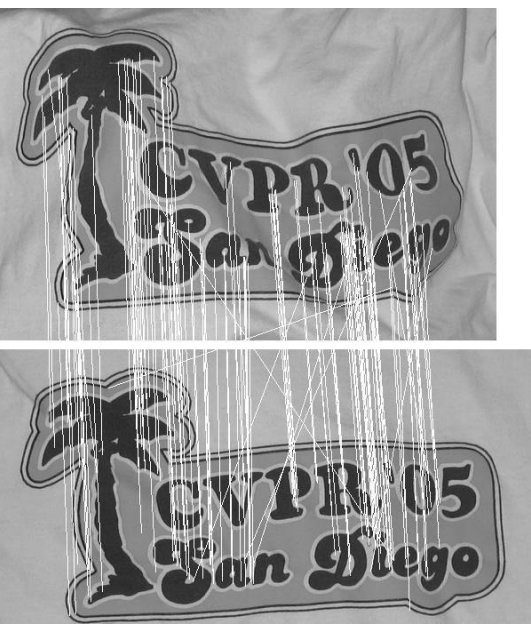

Hessian-Affine: 55 matches
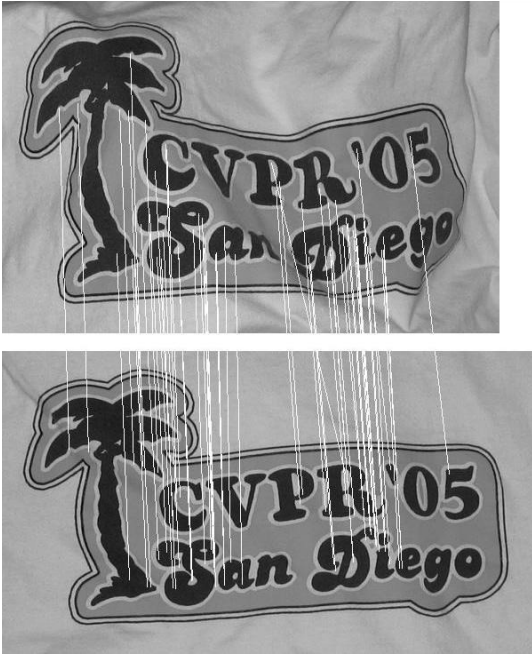

Figure 27: CVPR. Not shown: Harris-Affine (25 matches), MSER (17 matches).

ASIFT: 141 matches
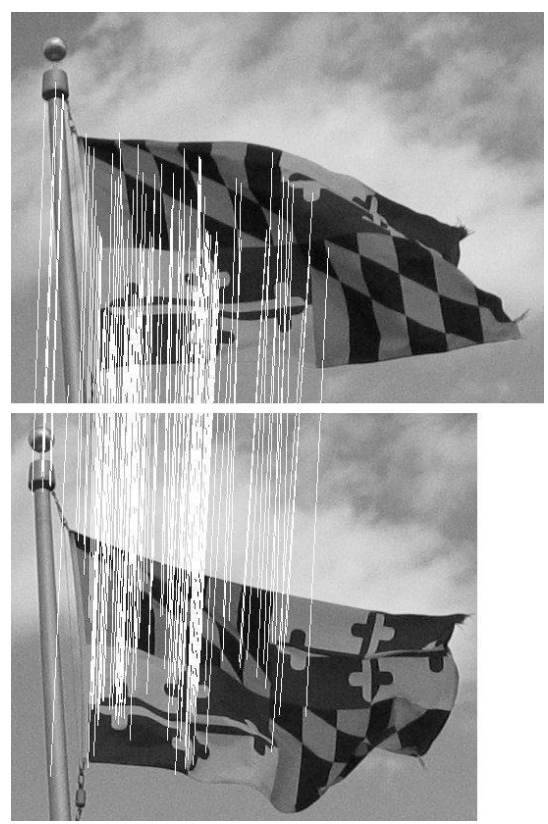

SIFT: 31 matches
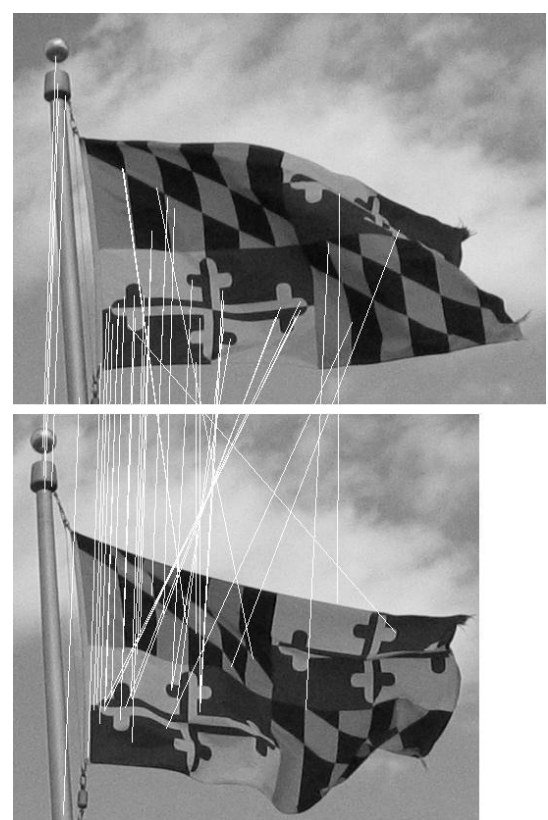

Harris-Affine: 15 matches
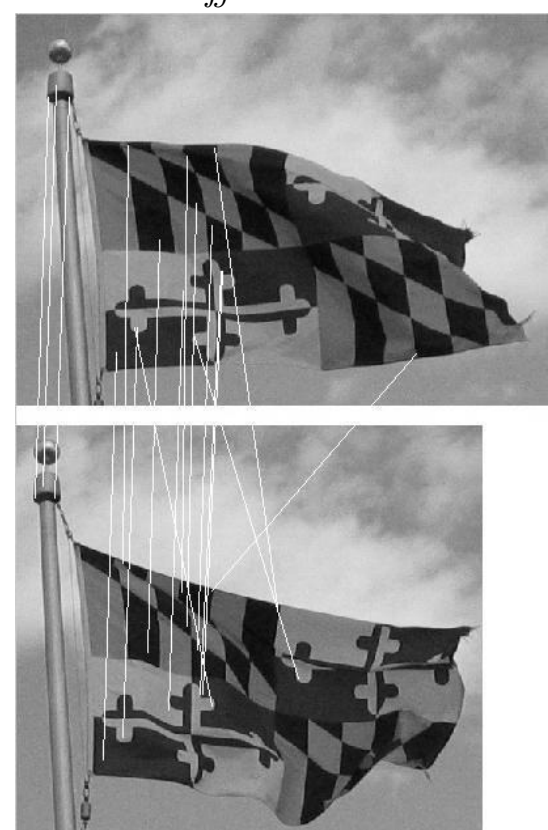

Figure 28: Flag. Not shown: Hessian-Affine (10 matches), MSER (2 matches). 

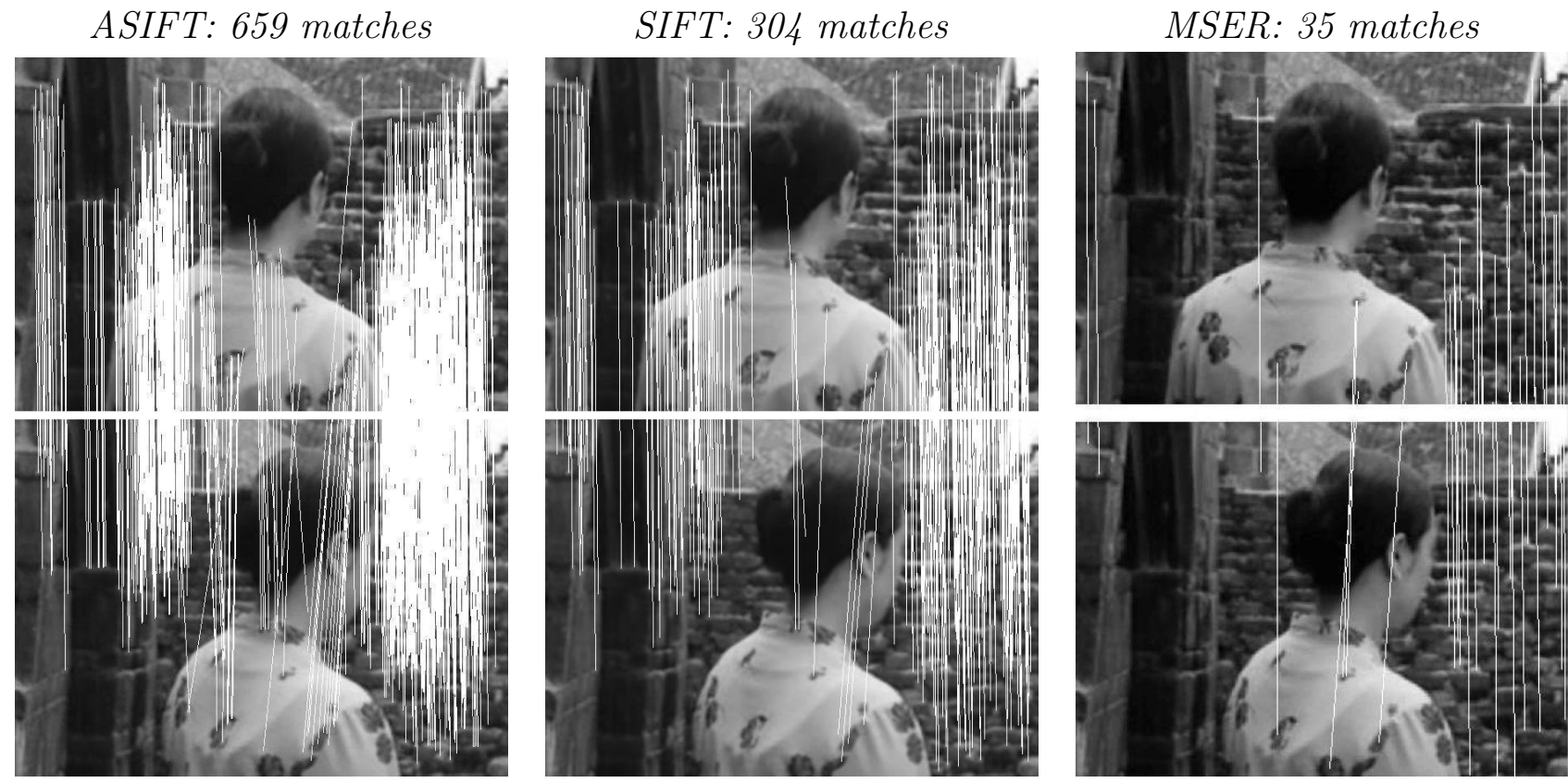

Figure 29: Girl. Not shown: Harris-Affine (45 matches, 1 on the cloth), Hessian-Affine (22 matches, 1 on the cloth). 
ASIFT: 33 matches

SIFT: 4 matches
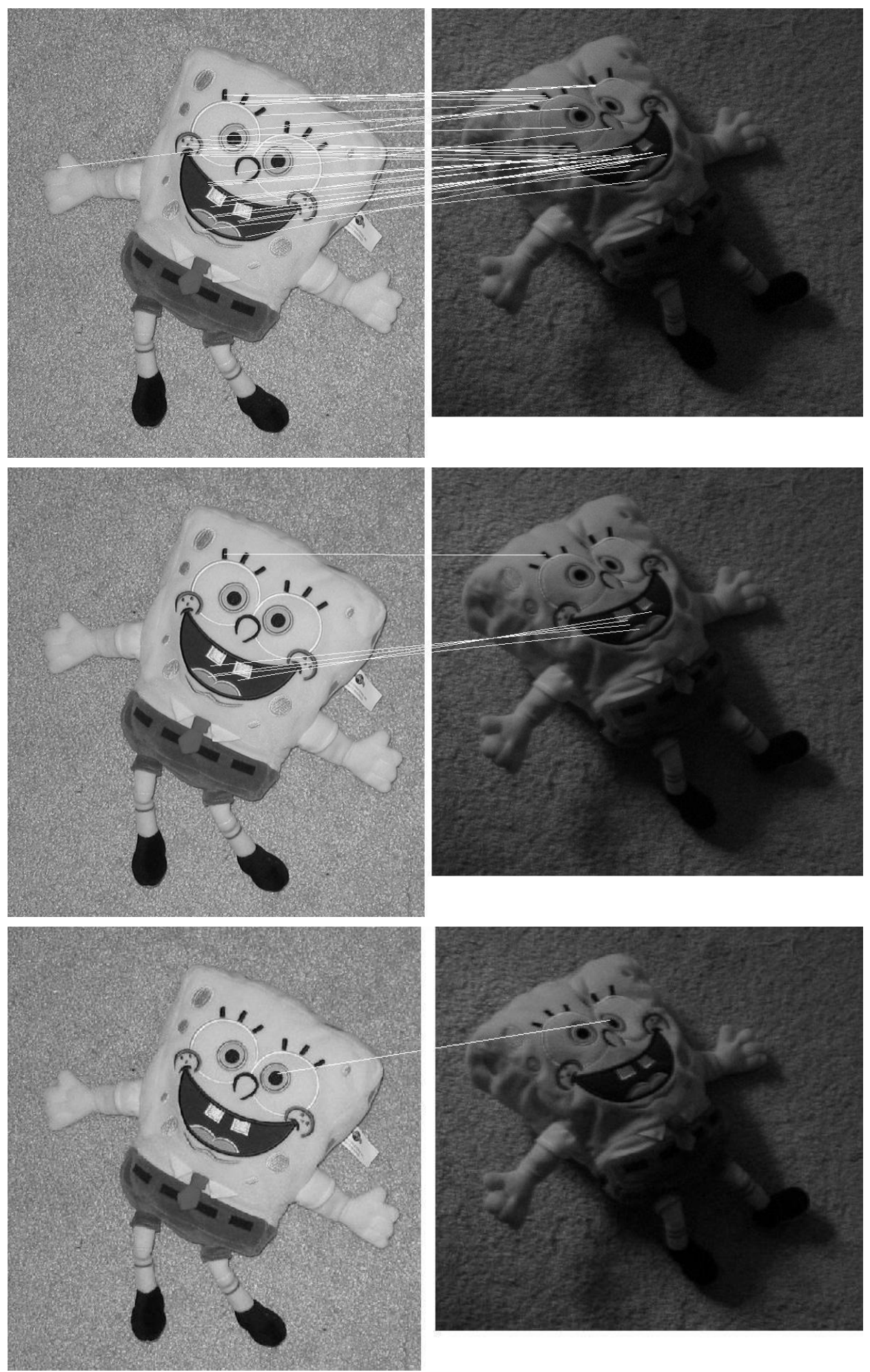

Hessian-Affine: 1 match

Figure 30: Toy. Not shown: Harris-Affine (0 match), MSER (0 match). 

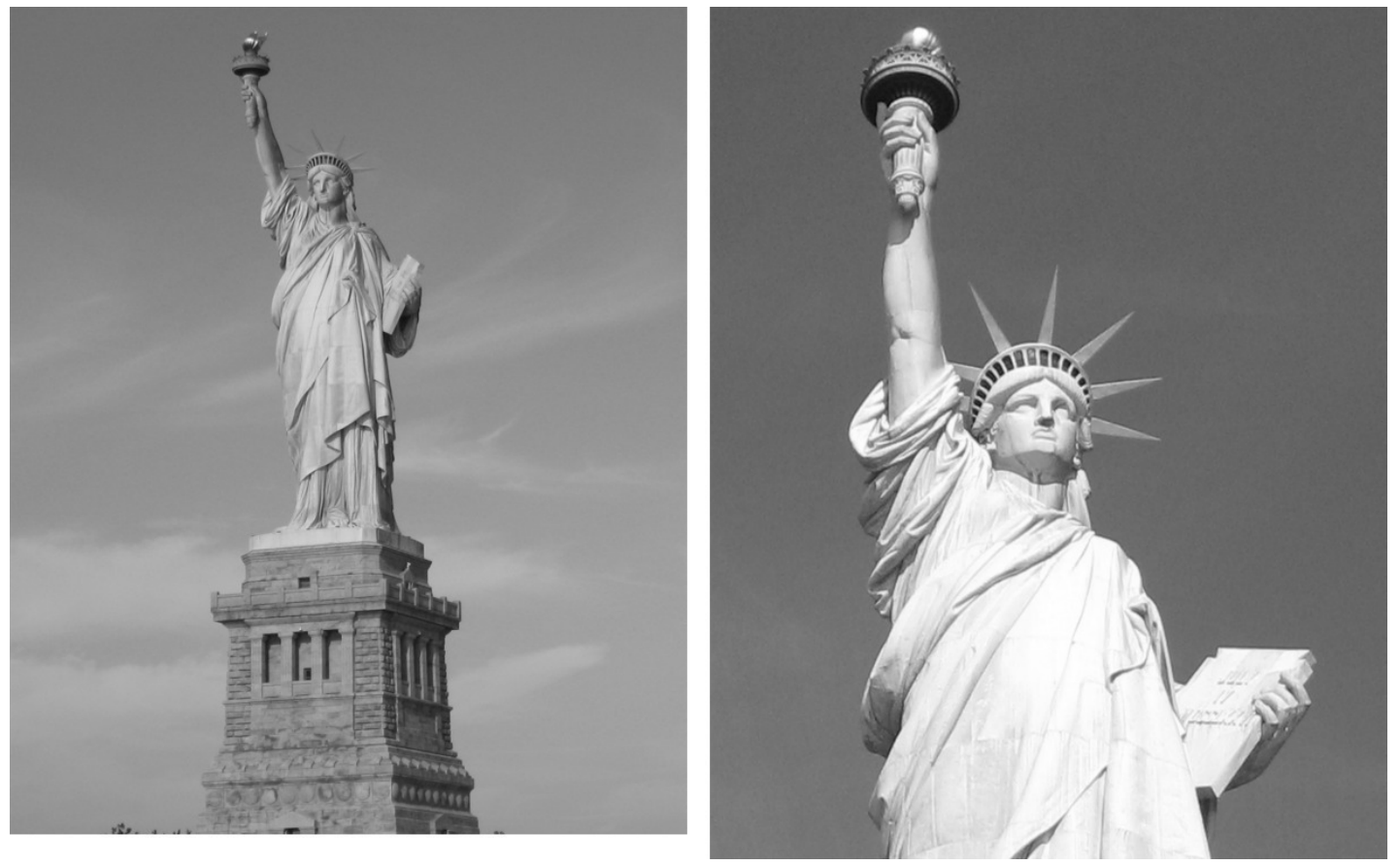

Figure 31: Strong relief effect. All methods fail! ASIFT, SIFT, Harris-Affine, Hessian-Affine, MSER: 0 match. The image on the right in close-up shows strong relief effect. 

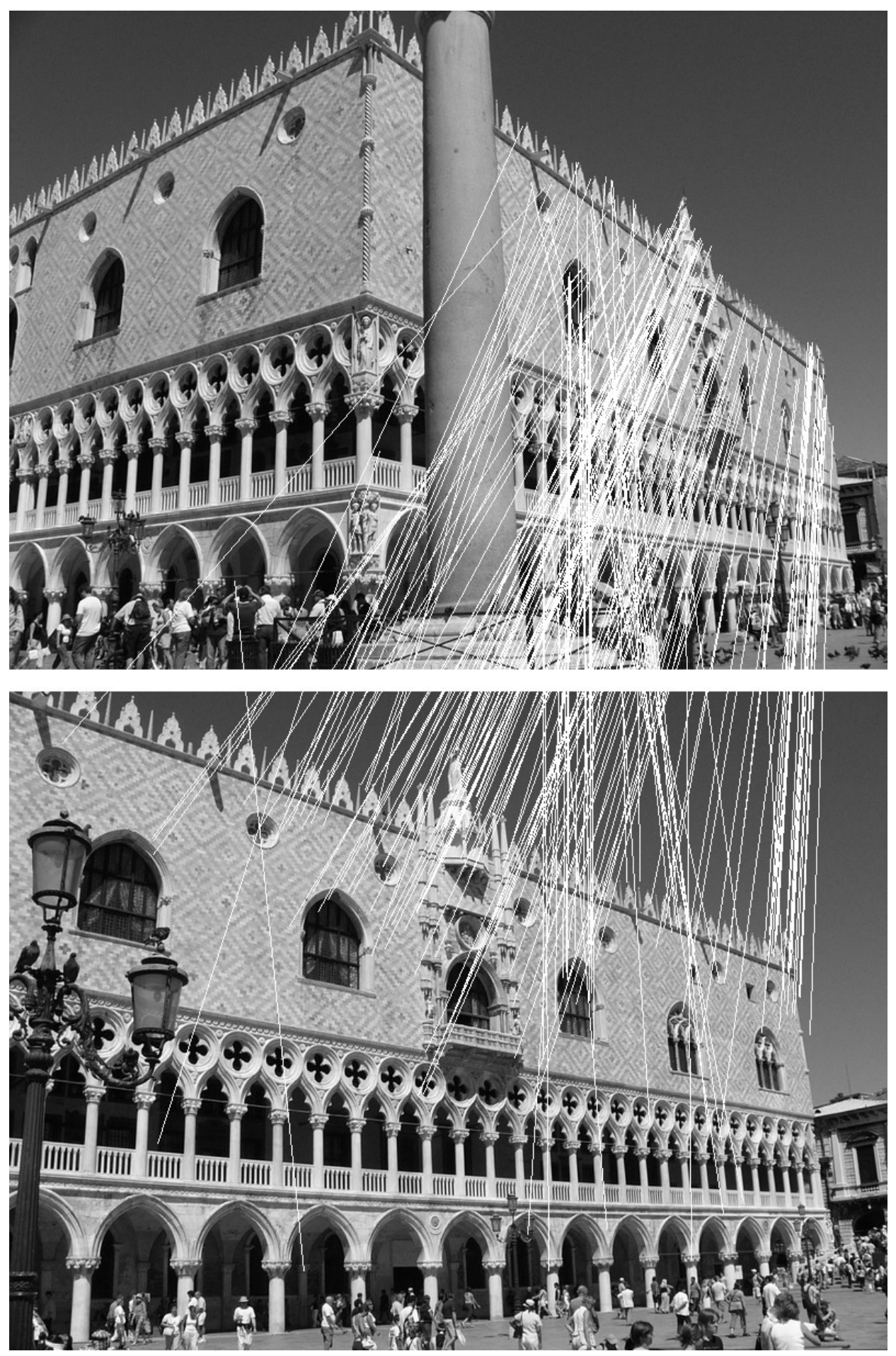

Figure 32: "Good" false matches. Matches can be arbitrary among repetitive shapes. ASIFT: 171 matches, many are "good" false matches (for example the matches between some windows). 


\section{Image credits}

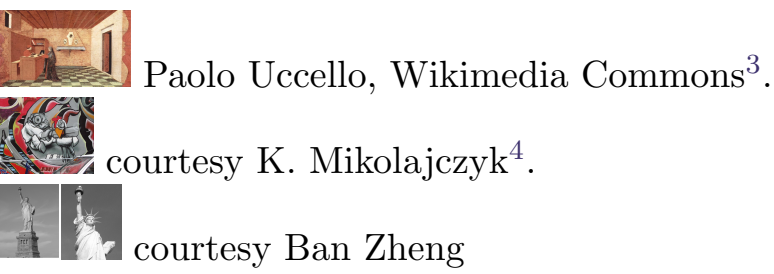

Object deformation images (Sponge Bob, CVPR, flag, girl, toy), courtesy Haibin Ling and David W. Jacobs ${ }^{5}$. All other images, (C) Guoshen Yu, CC-BY.

\section{References}

[1] D.G. Lowe. Distinctive image features from scale-invariant keypoints. International Journal of Computer Vision, 60(2):91-110, 2004. http://dx.doi.org/10.1023/B:VISI.0000029664. 99615.94.

[2] J. Matas, O. Chum, M. Urba, and T. Pajdla. Robust wide baseline stereo from maximally stable extremal regions. In Proceedings of British Machine Vision Conference, pages 384-396, 2002. http://dx.doi.org/10.1016/j.imavis.2004.02.006.

[3] K. Mikolajczyk and C. Schmid. An affine invariant interest point detector. In Proceedings of European Conference on Computer Vision, volume 2350, pages 128-142, 2002.

[4] K. Mikolajczyk, T. Tuytelaars, C. Schmid, A. Zisserman, J. Matas, F. Schaffalitzky, T. Kadir, and L. Van Gool. A comparison of affine region detectors. International Journal of Computer Vision, 65(1):43-72, 2005. http://dx.doi.org/10.1007/s11263-005-3848-x.

[5] L. Moisan and B. Stival. A probabilistic criterion to detect rigid point matches between two images and estimate the fundamental matrix. International Journal of Computer Vision, 57(3):201-218, 2004. http://dx.doi.org/10.1023/B:VISI.0000013094.38752.54.

[6] J.M. Morel and G.Yu. ASIFT: A New Framework for Fully Affine Invariant Image Comparison. SIAM Journal on Imaging Sciences, 2(2):438-469, 2011. http://dx.doi.org/10.1137/ 080732730.

[7] J.M. Morel and G.Yu. Is SIFT scale invariant? Inverse Problems and Imaging, 5(1):115-136, 2011. http://dx.doi.org/10.3934/ipi.2011.5.115.

[8] G. Yu and J.M. Morel. A fully affine invariant image comparison method. In Proceedings of IEEE International Conference on Acoustics, Speech, and Signal Processing (ICASSP), 2009. http://dx.doi.org/10.1109/ICASSP. 2009.4959904.

\footnotetext{
${ }^{3}$ http://fr.wikipedia.org/wiki/Fichier:Paolo_Uccello_062.jpg

${ }^{4}$ http://www.robots.ox.ac.uk/ vgg/research/affine/

${ }^{5}$ http://www.ist.temple.edu/ hbling/publication/ling-deformation-iccv05.pdf
} 